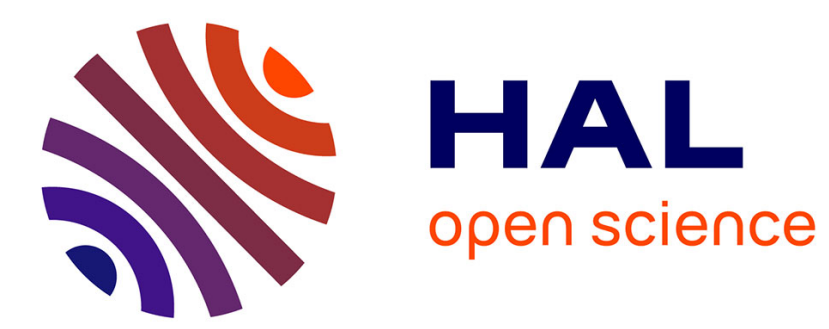

\title{
On the construction of wavelets on a bounded interval
}

Gerlind Plonka, Kathi Selig, Manfred Tasche

\section{To cite this version:}

Gerlind Plonka, Kathi Selig, Manfred Tasche. On the construction of wavelets on a bounded interval. Advances in Computational Mathematics, 1995, 4, pp.357-388. 10.1007/BF02123481 . hal-01322918

\section{HAL Id: hal-01322918 https://hal.science/hal-01322918}

Submitted on 28 May 2016

HAL is a multi-disciplinary open access archive for the deposit and dissemination of scientific research documents, whether they are published or not. The documents may come from teaching and research institutions in France or abroad, or from public or private research centers.
L'archive ouverte pluridisciplinaire HAL, est destinée au dépôt et à la diffusion de documents scientifiques de niveau recherche, publiés ou non, émanant des établissements d'enseignement et de recherche français ou étrangers, des laboratoires publics ou privés.

\section{다(1) (2)}

Distributed under a Creative Commons Attribution - ShareAlikel 4.0 International 


\title{
On the construction of wavelets on a bounded interval
}

\author{
Gerlind Plonka, Kathi Selig and Manfred Tasche \\ Fachbereich Mathematik, Universität Rostock, D-18051 Rostock, Germany
}

\begin{abstract}
This paper presents a general approach to a multiresolution analysis and wavelet spaces on the interval $[-1,1]$. Our method is based on the Chebyshev transform, corresponding shifts and the discrete cosine transformation (DCT). For the wavelet analysis of given functions, efficient decomposition and reconstruction algorithms are proposed using fast DCT-algorithms. As examples for scaling functions and wavelets, polynomials and transformed splines are considered.
\end{abstract}

\section{Introduction}

Recently, several constructions of wavelets on a bounded interval have been presented. Most of these approaches are based on the theory of cardinal wavelets. The simplest construction consists in the trivial extension of functions $f:[0,1] \rightarrow \mathbb{R}$ by setting $f(x):=0$ for $x \in \mathbb{R} \backslash[0,1]$. These functions can be analyzed by means of cardinal wavelets. But in general, this extension produces discontinuities at $x=0$ as well as $x=1$, which are reflected by large wavelet coefficients for high levels near the endpoints 0 and 1 , even if $f$ is smooth on $[0,1]$. Thus the regularity of $f$ is not characterized by the decay of wavelet coefficients.

Another simple solution, often adapted in image analysis, consists in the even 2-periodic extension $\tilde{f}$ of $f:[0,1] \rightarrow \mathbb{R}$. If $f \in C[0,1]$, then $\tilde{f} \in C(\mathbb{R})$. But in general, if $f \in C^{1}[0,1]$, then the derivative of $f$ has discontinuities at the integers. The smoothness of $f$ is again not characterized by the decay of wavelet coefficients.

In [8], Meyer has derived orthonormal wavelets on $[0,1]$ by restricting Daubechies' scaling functions and wavelets to $[0,1]$ and orthonormalizing their restrictions by the Gram-Schmidt procedure. This idea led to numerical instabilities such that further investigations of wavelets on a bounded interval were necessary (see [4]).

We are interested in wavelet methods on a bounded interval which can exactly analyze the boundary behaviour of given functions. Up to now, three methods are known to solve this problem. The often used first method is based on special 
boundary and interior scaling functions as well as wavelets (see $[3,4,13])$ such that numerical problems at the boundaries can be reduced. Then the bases of sample and wavelet spaces do not consist in shifts of single functions. The second method (see [9]) works with two generalized dilation operations, since the classical dilation is not applicable for functions on a bounded interval.

A third wavelet construction on the interval $I:=[-1,1]$, first proposed in [6], is based on Chebyshev polynomials. Both scaling functions and wavelets are polynomials which satisfy certain interpolation properties. As shown in [16], this polynomial wavelet approach can be considered as generalized version of the well-known wavelet concept, which is based on shift-invariant subspaces of the weighted Hilbert space $L_{w}^{2}(I)$ with respect to the Chebyshev shifts (see [2]), where $w$ denotes the Chebyshev weight.

The objective of this paper is a new general approach to multiresolution of $L_{w}^{2}(I)$ and to wavelets on the interval $I$, based on the ideas in [16]. As known, the Fourier transform and shift-invariant subspaces of $L^{2}(\mathbb{R})$ are essential tools for the construction of cardinal multiresolution and wavelets (see [5]). Analogously, the finite Fourier transform and shift-invariant subspaces of $L_{2 \pi}^{2}$ lead to a unified approach to periodic wavelets (see [7,11]). This concept can be transferred to the Hilbert space $L_{2 \pi, 0}^{2}$ of even periodic functions using the shift operator

$$
S_{a} F:=\frac{1}{2}(F(\cdot+a)+F(\cdot-a)) \quad(a \in \mathbb{R})
$$

for $F \in L_{2 \pi, 0}^{2}$. The isomorphism between $L_{2 \pi, 0}^{2}$ and $L_{w}^{2}(I)$ can be exploited in order to construct new sample and wavelet spaces in $L_{w}^{2}(I)$. Using fast algorithms of discrete cosine transforms (DCT), efficient frequency based algorithms for decomposition and reconstruction are proposed. As special scaling functions and wavelets, we consider algebraic polynomials and transformed splines. It is remarkable that our decomposition algorithm for polynomial wavelets needs fewer multiplications up to a certain level than the fast decomposition algorithm for cubic spline wavelets on $[0,1]$ proposed in [13].

The outline of our paper is as follows. In section 2 we briefly introduce the Chebyshev transform, related shifts and the DCT. In section 3 we analyze shiftinvariant subspaces of $L_{w}^{2}(I)$. The scalar product of functions from shift-invariant subspaces can be simplified to a finite sum by means of the so-called bracket product. In section 4 we consider a nonstationary multiresolution of $L_{w}^{2}(I)$ consisting of shift-invariant subspaces $V_{j}\left(j \in \mathbb{N}_{0}\right)$ generated by shifts of scaling functions $\varphi_{j}$. The required conditions for the multiresolution of $L_{w}^{2}(I)$ and their consequences for the scaling functions $\varphi_{j}$ are analyzed in detail. In section 5 we introduce the wavelet space $W_{j}\left(j \in \mathbb{N}_{0}\right)$ as the orthogonal complement of $V_{j}$ in $V_{j+1}$. Then $W_{j}$ is a shift-invariant subspace generated by shifts of the wavelet $\psi_{j}$. Using the two-scale symbol of $\varphi_{j}$ and the bracket product of $\varphi_{j}$ and $\varphi_{j+1}$, the wavelet $\psi_{j}$ is characterized in theorem 5.3. Section 6 provides fast, numerically stable decomposition and reconstruction algorithms based on fast DCT-algorithms. In section 7 we present polynomial wavelets on $I$ (see $[6,16])$. Finally in section 8 , we adapt the theory of periodic splines to the interval $I$ with respect to the Chebyshev 
nodes. Note that the transformed spline wavelets are supported on small subintervals of $I$. The examples show that periodic multiresolutions of $L_{2 \pi}^{2}$ with even scaling functions $\varphi_{j}$ can be transformed into a multiresolution of $L_{w}^{2}(I)$.

\section{Chebyshev transform and shifts}

In this section, we introduce the Chebyshev transform and corresponding shifts and we examine their relations to the even shifts of periodic even functions. For more details on Chebyshev shifts we refer to $[2,16]$. Throughout this paper, we consider the interval $I:=[-1,1]$ and the Chebyshev weight $w(x):=\left(1-x^{2}\right)^{-1 / 2}$ for $x \in(-1,1)$. Let $L_{w}^{2}(I)$ be the weighted Hilbert space of all measurable functions $f: I \rightarrow \mathbb{R}$ with the property

$$
\int_{l} w(y) f(y)^{2} \mathrm{~d} y<\infty
$$

For $f, g \in L_{w}^{2}(I)$, the corresponding inner product and norm are defined by

$$
\langle f, g\rangle:=\frac{2}{\pi} \int_{I} w(y) f(y) g(y) \mathrm{d} y, \quad\|f\|:=\langle f, f\rangle^{1 / 2} .
$$

Let $l^{2}$ denote the Hilbert space of all real, square summable sequences $\mathbf{a}=\left(a_{n}\right)_{n=0}^{\infty}$, $\mathbf{b}=\left(b_{n}\right)_{n=0}^{\infty}$ with the weighted inner product and norm given by

$$
(\mathbf{a}, \mathbf{b})_{l^{2}}:=\frac{1}{2} a_{0} b_{0}+\sum_{n=1}^{\infty} a_{n} b_{n}, \quad\|\mathbf{a}\|_{l^{2}}:=(\mathbf{a}, \mathbf{a})_{\ell^{2}}^{1 / 2} .
$$

Let $C(I)$ be the set of all continuous functions $f: I \rightarrow \mathbb{R}$. By $\Pi_{n}\left(n \in \mathbb{N}_{0}\right)$ we denote the set of all real polynomials of degree at most $n$ restricted on $I$. As known, the Chebyshev polynomials $T_{n}:=\cos (n \arccos ) \in \Pi_{n}\left(n \in \mathbb{N}_{0}\right)$ form a complete orthogonal system in $L_{w}^{2}(I)$. Note that arccos $: I \rightarrow[0, \pi]$ is the inverse function of cos restricted on $[0, \pi]$. For $m, n \in \mathbb{N}_{0}$ we have

$$
\left\langle T_{m}, T_{n}\right\rangle= \begin{cases}2 & m=n=0, \\ 1 & m=n>0, \\ 0 & m \neq n\end{cases}
$$

Further, we use the Chebyshev transform of $L_{w}^{2}(I)$ into $l^{2}$ mapping $f \in L_{w}^{2}(I)$ into $\mathbf{a}[f]:=\left(a_{n}[f]\right)_{n=0}^{\infty} \in l^{2}$ with the Chebyshev coefficients

$$
a_{n}[f]:=\left\langle f, T_{n}\right\rangle \quad\left(n \in \mathbb{N}_{0}\right) .
$$

Then for $f, g \in L_{w}^{2}(I)$, we have the Parseval identities

$$
\langle f, g\rangle=(\mathbf{a}[f], \mathbf{a}[g])_{1^{2}}, \quad\|f\|=\|\mathbf{a}[f]\|_{1^{2}} .
$$

Note that the Chebyshev transform is a linear bijective mapping of $L_{w}^{2}(I)$ onto $l^{2}$. For more details on the Chebyshev transform see $[2,10]$. 
The Chebyshev transform is strongly related with the Fourier cosine transform. Let $L_{2 \pi}^{2}$ be the Hilbert space of all $2 \pi$-periodic, square integrable functions $F, G: \mathbb{R} \rightarrow \mathbb{R}$ with the inner product

$$
(F, G)_{2}:=\frac{1}{2 \pi} \int_{-\pi}^{\pi} F(s) G(s) \mathrm{d} s .
$$

Let $L_{2 \pi, 0}^{2}$ be the subspace of all even functions of $L_{2 \pi}^{2}$. For a given function $f \in L_{w}^{2}(I)$, the cos-transformed function $F:=f(\cos ) \in L_{2 \pi, 0}^{2}$ has the Fourier expansion

$$
F=\frac{1}{2} a_{0}(F)+\sum_{n=1}^{\infty} a_{n}(F) \cos (n \cdot)
$$

with the Fourier cosine coefficients

$$
a_{n}(F):=\frac{2}{\pi} \int_{0}^{\pi} F(s) \cos (n s) \mathrm{d} s \quad\left(n \in \mathbb{N}_{0}\right) .
$$

In order to adapt the concept of shifts to the interval $I$, we consider the even shift $S_{a} F$ of $F \in L_{2 \pi, 0}^{2}$ by $a \in \mathbb{R}$, which is defined as the even part of the translated function $F(\cdot-a)$, i.e.

$$
S_{a} F:=\frac{1}{2}(F(\cdot+a)+F(\cdot-a)) \in L_{2 \pi, 0}^{2} .
$$

Observe that for $n \in \mathbb{N}_{0}$

$$
S_{a} \cos (n \cdot)=\cos (n a) \cos (n \cdot), \quad a_{n}\left(S_{a} F\right)=\cos (n a) a_{n}(F) .
$$

Restricting $F=f(\cos )$ on $[0, \pi]$, the arccos-transformed function $F(\arccos )$ coincides with $f \in L_{w}^{2}(I)$. From (2.2)-(2.3) it follows directly the Chebyshev expansion

$$
f=\frac{1}{2} a_{0}[f]+\sum_{n=1}^{\infty} a_{n}[f] T_{n}, \quad a_{n}[f]=a_{n}(f(\cos )) \quad\left(n \in \mathbb{N}_{0}\right) .
$$

Further, the even shift $S_{a}$ of $F=f(\cos )(a \in \mathbb{R})$ goes into the Chebyshev shift $s_{h} f$ of $f$ with $h:=\cos a \in I$, i.e.

$$
\left(s_{h} f\right)(x):=\frac{1}{2} f(x h-v(x) v(h))+\frac{1}{2} f(x h+v(x) v(h)) \quad(x \in I)
$$

with $v(x):=\left(1-x^{2}\right)^{1 / 2}(x \in I)$.

For the realization of the Chebyshev transform in finite dimensional subspaces of $L_{w}^{2}(I)$, we will use fast algorithms of the discrete cosine transform (DCT). In the following, we briefly introduce the different types of DCT.

Let $N_{j}:=d 2^{j}$, where $j \in \mathbb{N}_{0}$ stands for the level and $d \in \mathbb{N}$ is a constant depending on the application. Further, let $\delta_{k, l}$ be the Kronecker symbol and $\epsilon_{j, 0}=\epsilon_{j, N_{j}}:=2^{-1}$, $\epsilon_{j, k}:=1\left(k=1, \ldots, N_{j}-1\right)$. We introduce the matrices

$$
\begin{array}{lll}
\mathbf{C}_{j}:=\left(\cos \frac{k l \pi}{N_{j}}\right)_{k, l=0}^{N_{j}}, & \mathbf{D}_{j}:=\operatorname{diag}\left(\epsilon_{j, k}\right)_{k=0}^{N_{j}}, & \mathbf{I}_{j}:=\left(\delta_{k, l}\right)_{k, l=0}^{N_{j}}, \\
\tilde{\mathbf{C}}_{j}:=\left(\cos \frac{(2 s+1) r \pi}{N_{j+1}}\right)_{r, s=0}^{N_{j}-1}, & \tilde{\mathbf{D}}_{j}:=\operatorname{diag}\left(\epsilon_{j, s}\right)_{s=0}^{N_{j}-1}, & \tilde{\mathbf{I}}_{j}:=\left(\delta_{r, s}\right)_{r, s=0}^{N_{j-1},},
\end{array}
$$


which fulfil the relations

$$
\begin{array}{r}
\mathbf{C}_{j} \mathbf{D}_{j} \mathbf{C}_{j} \mathbf{D}_{j}=\frac{N_{j}}{2} \mathbf{I}_{j}, \\
\tilde{\mathbf{C}}_{j}^{\mathrm{T}} \tilde{\mathbf{D}}_{j} \tilde{\mathbf{C}}_{j}=\tilde{\mathbf{C}}_{j} \tilde{\mathbf{C}}_{j}^{\mathrm{T}} \tilde{\mathbf{D}}_{j}=\frac{N_{j}}{2} \tilde{\mathbf{I}}_{j} .
\end{array}
$$

This follows from

$$
\begin{gathered}
\sum_{k=0}^{N_{j}} \epsilon_{j, k} \cos \frac{k u \pi}{N_{j}}= \begin{cases}N_{j} & u \equiv 0 \bmod N_{j+1}, \\
0 & \text { otherwise, }\end{cases} \\
\sum_{k=0}^{N_{j}-1} \cos \frac{(2 k+1) u \pi}{N_{j+1}}= \begin{cases}N_{j} & u \equiv 0 \bmod N_{j+2}, \\
-N_{j} & u \equiv N_{j+1} \bmod N_{j+2}, \\
0 & \text { otherwise }\end{cases}
\end{gathered}
$$

(cf. [16]). For further development, we define some variants of the DCT. The type I-DCT of length $N_{j}+1$ (DCT-I $\left(N_{j}+1\right)$ ) is a mapping of $\mathbb{R}^{N_{j}+1}$ into itself defined by

$$
\hat{\mathbf{x}}^{\mathrm{I}}:=\mathbf{C}_{j}^{\mathrm{I}} \mathbf{x} \quad\left(\mathbf{x} \in \mathbb{R}^{N_{j}+1}\right)
$$

with $\mathbf{C}_{j}^{\mathrm{I}}:=\mathbf{C}_{j} \mathbf{D}_{j}$. By $N_{j}\left(\mathbf{C}_{j}^{\mathrm{I}}\right)^{-1}=2 \mathbf{C}_{j}^{\mathrm{I}}$, this mapping is bijective. Note that (2.6) and (2.10) imply

$$
\left(\hat{\mathbf{x}}^{\mathrm{I}}\right)^{\mathrm{T}} \mathbf{D}_{j} \hat{\mathbf{x}}^{\mathrm{I}}=\mathbf{x}^{\mathrm{T}}\left(\mathbf{C}_{j}^{\mathrm{I}}\right)^{\mathrm{T}} \mathbf{D}_{j} \mathbf{C}_{j}^{\mathrm{I}} \mathbf{x}=\mathbf{x}^{\mathrm{T}} \mathbf{D}_{j} \mathbf{C}_{j} \mathbf{D}_{j} \mathbf{C}_{j} \mathbf{D}_{j} \mathbf{x}=\frac{N_{j}}{2} \mathbf{x}^{\mathrm{T}} \mathbf{D}_{j} \mathbf{x} .
$$

The type II-DCT of length $N_{j}$ (DCT-II $\left(N_{j}\right)$ ) is a mapping of $\mathbb{R}^{N_{j}}$ into itself defined by

$$
\tilde{\mathbf{y}}^{\mathrm{II}}:=\mathbf{C}_{j}^{\mathrm{II}} \mathbf{y} \quad\left(\mathbf{y} \in \mathbb{R}^{N_{j}}\right)
$$

with $\mathbf{C}_{j}^{\mathrm{II}}:=\tilde{\mathbf{C}}_{j}$. Then by (2.7) and (2.12), we obtain

$$
\left(\tilde{\mathbf{y}}^{\mathrm{II}}\right)^{\mathrm{T}} \tilde{\mathbf{D}}_{j} \tilde{\mathbf{y}}^{\mathrm{II}}=\mathbf{y}^{\mathrm{T}} \tilde{\mathbf{C}}_{j}^{\mathrm{T}} \tilde{\mathbf{D}}_{j} \tilde{\mathbf{C}}_{j} \mathbf{y}=\frac{N_{j}}{2} \mathbf{y}^{\mathrm{T}} \mathbf{y} .
$$

The type III-DCT of length $N_{j}\left(\mathrm{DCT}\right.$-III $\left.\left(N_{j}\right)\right)$ is a mapping of $\mathbb{R}^{N_{j}}$ into itself defined by

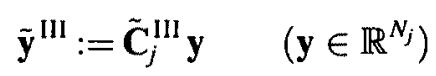

with $\mathbf{C}_{j}^{\mathrm{III}}:=\tilde{C}_{j}^{\mathrm{T}} \tilde{\mathbf{D}}_{j}$. By (2.7), the inverse of the DCT-II $\left(N_{j}\right)$ is the mapping $\left(2 / N_{j}\right)$ DCT-III $\left(N_{j}\right)$. Fast and numerically stable algorithms for the DCT-I $\left(N_{j}+1\right)$, DCT-II $\left(N_{j}\right)$ and DCT-III $\left(N_{j}\right)$, which work in real arithmetic, are described in $[1,15]$. 


\section{Shift-invariant subspaces}

Using the Gauss-Chebyshev nodes $h_{j, u}:=\cos \left(u \pi / N_{j}\right)(u \in \mathbb{Z})$ of level $j\left(j \in \mathbb{N}_{0}\right)$ and the Chebyshev shift (2.5), we obtain the shifts of level $j$

$$
\sigma_{j, u}:=s_{h j, u} \quad(u \in \mathbb{Z}),
$$

which possess the following properties (see $[2,16]$ ):

\section{Lemma 3.1}

For $j \in \mathbb{N}_{0}, u, v \in \mathbb{Z}$ and $f, g \in L_{w}^{2}(I)$ we have

(i) $\sigma_{j, u+N_{j+1}}=\sigma_{j, \pm u}=\sigma_{j+1,2 u}$,

(ii) $2 \sigma_{j, u} \sigma_{j, v}=2 \sigma_{j, v} \sigma_{j, u}=\sigma_{j, u+v}+\sigma_{j, u-v}$,

(iii) $\left\langle\sigma_{j, u} f, g\right\rangle=\left\langle f, \sigma_{j, u} g\right\rangle$,

(iv) $\sigma_{j, u} T_{n}=\cos \left(n u \pi / N_{j}\right) T_{n}, a_{n}\left[\sigma_{j, u} f\right]=\cos \left(n u \pi / N_{j}\right) a_{n}[f]\left(n \in \mathbb{N}_{0}\right)$,

(v) $\sigma_{j, u} f \in \Pi_{n}$ for $f \in \Pi_{n}\left(n \in \mathbb{N}_{0}\right)$. have

Note that $\sigma_{j, 0} f=f$ and $\sigma_{j, N_{j}} f=f(-\cdot)$ for $f \in L_{w}^{2}(I)$. Further, for $f \in C(I)$ we

$$
\left(\sigma_{j, u} f\right)(1)=f\left(h_{j, u}\right) \quad(u \in \mathbb{Z}) .
$$

A linear subspace $S$ of $L_{w}^{2}(I)$ is called shift-invariant of level $j\left(j \in \mathbb{N}_{0}\right)$, if for each $f \in S$ all shifted functions $\sigma_{j, l} f\left(l=0, \ldots, N_{j}\right)$ are contained in $S$. The shift-invariant subspace of level $j$

$$
S_{j, 0}(\varphi):=\operatorname{span}\left\{\sigma_{j, l} \varphi: l=0, \ldots, N_{j}\right\}
$$

is said to be of type 0 generated by $\varphi \in L_{w}^{2}(I)$. The shift-invariant subspace of level $j$

$$
S_{j, 1}(\varphi):=\operatorname{span}\left\{\sigma_{j+1,2 l+1} \varphi: l=0, \ldots, N_{j}-1\right\}
$$

is said to be of type 1 generated by $\varphi \in L_{w}^{2}(I)$. It is obvious by lemma 3.1, (i)-(ii) that $S_{j, 0}(\varphi) \subseteq S_{j+1,0}(\varphi)$ and $S_{j, 1}(\varphi)=S_{j, 0}\left(\sigma_{j+1,1} \varphi\right) \subseteq S_{j+1,0}(\varphi)$. By definition, $f \in S_{j+1,0}(\varphi)$ can be represented in the form

$$
f=\sum_{k=0}^{N_{j+1}} \epsilon_{j+1, k} \alpha_{j+1, k}(f) \sigma_{j+1, k} \varphi \quad\left(\alpha_{j+1, k}(f) \in \mathbb{R}\right) .
$$

Using lemma 3.1, (iv) and Chebyshev transform, we obtain the Chebyshev coefficients

$$
a_{n}[f]=\hat{\alpha}_{j+1, n}(f) a_{n}[\varphi] \quad\left(n \in \mathbb{N}_{0}\right)
$$

with

$$
\hat{\alpha}_{j+1, n}(f):=\sum_{k=0}^{N_{j+1}} \epsilon_{j+1, k} \alpha_{j+1, k}(f) \cos \frac{k n \pi}{N_{j+1}} .
$$

Observe that $\left(\hat{\alpha}_{j+1, n}(f)\right)_{n=0}^{N_{j+1}}$ is the DCT-I $\left(N_{j+1}+1\right)$ of $\left(\alpha_{j+1, k}(f)\right)_{k=0}^{N_{j+1}}$ and that the 
following properties of periodicity and symmetry hold for $n \in \mathbb{N}_{0}$ and $k=0, \ldots, N_{j+1}-1$

$$
\hat{\alpha}_{j+1, n}(f)=\hat{\alpha}_{j+1, N_{j+2}+n}(f), \quad \hat{\alpha}_{j+1, k}(f)=\hat{\alpha}_{j, N_{j+2}-k}(f) .
$$

In particular, for $f \in S_{j, 0}(\varphi)$ we get the representation (3.2) with

$$
\alpha_{j+1,2 l+1}(f):=0 \quad\left(l=0, \ldots, N_{j}-1\right) .
$$

Then it follows that the vector $\left(\hat{\alpha}_{j+1, n}(f)\right)_{n=0}^{N_{j}}$ with components (3.4) is the DCT-I $\left(N_{i}+1\right)$ of $\left(\alpha_{j+1,2 l}(f)\right)_{l=0}^{N_{j}}$. For $f \in S_{j, 1}(\varphi)$ we obtain (3.2) with

$$
\alpha_{j+1,2 l}(f):=0 \quad\left(l=0, \ldots, N_{j}\right),
$$

and the corresponding vector $\left(\hat{\alpha}_{j+1, n}(f)\right)_{n=0}^{N_{j}-1}$ is the $\operatorname{DCT}-\operatorname{II}\left(N_{j}\right)$ of the vector $\left(\alpha_{j+1,2 l+1}(f)\right)_{l=0}^{N_{j}-1}$, i.e.

$$
\hat{\alpha}_{j+1, n}(f)=\sum_{l=0}^{N_{j}-1} \alpha_{j+1,2 l+1}(f) \cos \frac{(2 l+1) n \pi}{N_{j+1}} .
$$

In the following, we derive some important properties of the subspaces $S_{j, \nu}(\varphi)$. We characterize $S_{j, \nu}(\varphi)(\nu \in\{0,1\})$ by the Chebyshev transform:

\section{Lemma 3.2}

Let $j \in \mathbb{N}_{0}, \nu \in\{0,1\}$ and $\varphi, f \in L_{w}^{2}(I)$ be given.

(i) Then $f \in S_{j, \nu}(\varphi)$ if and only if there exist $\hat{\alpha}_{j+1, n}(f) \in \mathbb{R}\left(n \in \mathbb{N}_{0}\right)$ with

$$
\begin{aligned}
\hat{\alpha}_{j+1, n}(f) & =\hat{\alpha}_{j+1, N_{j+2}+n}(f) & & \left(n \in \mathbb{N}_{0}\right), \\
\hat{\alpha}_{j+1, N_{j+1} \pm n}(f) & =(-1)^{\nu} \hat{\alpha}_{j+1, n}(f) & & \left(n=0, \ldots, N_{j}\right),
\end{aligned}
$$

such that (3.3) is satisfied.

(ii) Let $\sigma_{j+1, \nu} f \in S_{j, \nu}(\varphi)$. Then $S_{j, \nu}(f)=S_{j, \nu}(\varphi)$ if and only if

$$
\operatorname{supp} \mathbf{a}\left[\sigma_{j+1, \nu} f\right]=\operatorname{supp} \mathbf{a}\left[\sigma_{j+1, \nu} \varphi\right] \text {, }
$$

where supp $\mathbf{a}[f]:=\left\{n \in \mathbb{N}_{0}: a_{n}[f] \neq 0\right\}$ is the support of $\mathbf{a}[f]$.

\section{Proof}

As mentioned before, if $f \in S_{j, \nu}(\varphi)$, then (3.7) is satisfied. Since the Chebyshev transform is a linear bijective mapping, the proof of the reversed direction is straightforward. Hence (i) is valid. Now we show (ii) for $\nu=0$.

1) If $S_{j, 0}(f)=S_{j, 0}(\varphi)$, then $\varphi \in S_{j, 0}(f)$. From (i) it follows that supp $\mathbf{a}[\varphi] \subseteq$ supp a $[f]$. Analogously, by $f \in S_{j, 0}(\varphi)$ we find suppa $[f] \subseteq \operatorname{suppa}[\varphi]$. Hence we obtain (3.8).

2) Assume that (3.8) is satisfied. We only need to show that $\varphi \in S_{j, 0}(f)$. Since $f \in S_{j, 0}(\varphi)$, we have (3.3) with (3.7). By supp $\mathbf{a}[f]=\operatorname{supp} \mathbf{a}[\varphi]$, we conclude that 
$a_{n}[\varphi]=\hat{\beta}_{j+1, n} a_{n}[f]\left(n \in \mathbb{N}_{0}\right)$ with

$$
\hat{\beta}_{j+1, n}:= \begin{cases}\hat{\alpha}_{j+1, n}(f)^{-1} & \text { if } \hat{\alpha}_{j+1, n}(f) \neq 0, \\ 0 & \text { otherwise, }\end{cases}
$$

for which (3.7) is also satisfied.

For $\nu=1$, the assertion follows immediately from $S_{j, 1}(\varphi)=S_{j, 0}\left(\sigma_{j+1,1} \varphi\right)$ and $S_{j, 1}(f)=S_{j, 0}\left(\sigma_{j+1,1} f\right)$.

For a further analysis of the shift-invariant subspaces of $L_{w}^{2}(I)$, we introduce the bracket product of $\mathbf{a}:=\left(a_{n}\right)_{n=0}^{\infty}$ and $\mathbf{b}:=\left(b_{n}\right)_{n=0}^{\infty} \in l^{2}$. Let for $k=0, \ldots, N_{j+1}$

$$
[\mathbf{a}, \mathbf{b}]_{j, k}:=\sum_{m=0}^{\infty}\left(a_{m N_{j+1}+k} b_{m N_{j+1}+k}+a_{(m+1) N_{j+1}-k} b_{(m+1) N_{j+1}-k}\right) .
$$

Observe that $[\mathbf{a}, \mathbf{b}]_{j, k}$ satisfies the symmetry property

$$
[\mathbf{a}, \mathbf{b}]_{j, N_{j+1}-l}=[\mathbf{a}, \mathbf{b}]_{j, l} \quad\left(l=0, \ldots, N_{j+1}-1\right) .
$$

We extend the values $[\mathbf{a}, \mathbf{b}]_{j, k}\left(k=0, \ldots, N_{j+1}\right)$ to an $N_{j+1}$-periodic sequence, i.e.

$$
[\mathbf{a}, \mathbf{b}]_{j, k}=[\mathbf{a}, \mathbf{b}]_{j, k+N_{j+1}} \quad\left(k \in \mathbb{N}_{0}\right) .
$$

Then the type I-bracket product of length $N_{j}+1$ is defined by

$$
[\mathbf{a}, \mathbf{b}]_{j}^{\mathrm{I}}:=\left([\mathbf{a}, \mathbf{b}]_{j, k}\right)_{k=0}^{N_{j}} \in \mathbb{R}^{N_{j}+1},
$$

and the type II-bracket product of length $N_{j}$ by

$$
[\mathbf{a}, \mathbf{b}]_{j}^{\mathrm{II}}:=\left([\mathbf{a}, \mathbf{b}]_{j, k}\right)_{k=0}^{N_{j}-1} \in \mathbb{R}^{N_{j}} .
$$

\section{Lemma 3.3}

Let $j \in \mathbb{N}_{0}, \nu, \mu \in\{0,1\}$ and $\varphi, \psi \in L_{w}^{2}(I)$ be given. Further, let $f \in S_{j, \nu}(\varphi)$, $g \in S_{j, \mu}(\psi)$ with

$$
a_{n}[f]=\hat{\alpha}_{j+1, n}(f) a_{n}[\varphi], \quad a_{n}[g]=\hat{\beta}_{j+1, n}(g) a_{n}[\psi] \quad\left(n \in \mathbb{N}_{0}\right)
$$

be given, where $\hat{\alpha}_{j+1, n}(f), \hat{\beta}_{j+1, n}(g) \in \mathbb{R}$ possess the properties (3.7). Then we have

$$
\left.\langle f, g\rangle=\sum_{k=0}^{N_{j+1}} \epsilon_{j+1, k} \hat{\alpha}_{j+1, k}(f) \hat{\beta}_{j+1, k}(g)[\mathbf{a}[\varphi], \mathbf{a} \mid \psi]\right]_{j+1, k} .
$$

In particular, for $\mu=\nu$,

$$
\langle f, g\rangle=\sum_{k=0}^{N_{j}-\nu} \epsilon_{j, k} \hat{\alpha}_{j+1, k}(f) \hat{\beta}_{j+1, k}(g)[\mathbf{a}[\varphi], \mathbf{a}[\psi]]_{j, k} .
$$

Using the Parseval identity (2.1), the proof follows by straightforward calculations. In particular, with $f:=\sigma_{j, l} \varphi, g:=\sigma_{j, m} \psi$ for arbitrary $\varphi, \psi \in L_{w}^{2}(I)$, we 
obtain for $l, m=0, \ldots, N_{j}$ the relations

$$
\left\langle\sigma_{j, 1} \varphi, \sigma_{j, m} \psi\right\rangle=\sum_{k=0}^{N_{j}} \epsilon_{j, k} \cos \frac{k l \pi}{N_{j}} \cos \frac{k m \pi}{N_{j}}[\mathbf{a}[\varphi], \mathbf{a}[\psi]]_{j, k},
$$

i.e.,

$$
\left(\left\langle\sigma_{j, l} \varphi, \sigma_{j, m} \psi\right\rangle\right)_{l, m=0}^{N_{j}}=\mathbf{C}_{j} \mathbf{D}_{j} \operatorname{diag}[\mathbf{a}[\varphi], \mathbf{a}[\psi]]_{j}^{\mathrm{l}} \mathbf{C}_{j} .
$$

Analogously, for $f:=\sigma_{j+1,2 l+1} \varphi, g:=\sigma_{j+1,2 m+1} \psi$ we have for $l, m=0, \ldots, N_{j}-1$

$$
\left\langle\sigma_{j+1,2 l+1} \varphi, \sigma_{j+1,2 m+1} \psi\right\rangle=\sum_{k=0}^{N_{j}-1} \epsilon_{j, k} \cos \frac{k(2 l+1) \pi}{N_{j+1}} \cos \frac{k(2 m+1) \pi}{N_{j+1}}[\mathbf{a}[\varphi], \mathbf{a}[\psi]]_{j, k},
$$

and thus

$$
\left(\left\langle\sigma_{j+1,2 l+1} \varphi, \sigma_{j+1,2 m+1} \psi\right\rangle\right)_{l_{, m=0}}^{N_{j}-1}=\tilde{\mathbf{C}}_{j}^{\mathrm{T}} \tilde{\mathbf{D}}_{j} \operatorname{diag}[\mathbf{a}[\varphi], \mathbf{a}[\psi]]_{j}^{\mathrm{II}} \tilde{\mathbf{C}}_{j}
$$

\section{Corollary 3.4}

For $j \in \mathbb{N}_{0}$ and $\varphi, \psi \in L_{w}^{2}(I)$, we have

(i) $S_{j, \nu}(\varphi) \perp S_{j, \nu}(\psi)(\nu \in\{0,1\})$ if and only if

$$
[\mathbf{a}[\varphi], \mathbf{a}[\psi]]_{j, k}=0 \quad\left(k=0, \ldots, N_{j}-\nu\right) ;
$$

(ii) $S_{j, 0}(\varphi) \perp S_{j, 1}(\psi)$ if and only if

$$
[\mathbf{a}[\varphi], \mathbf{a}[\psi]]_{j+1, k}=0 \quad\left(k=0, \ldots, N_{j+1}\right) .
$$

For $\varphi \in L_{w}^{2}(I)$, we consider the system $\mathcal{B}_{j, 0}(\varphi):=\left\{\sigma_{j, l} \varphi: l=0, \ldots, N_{j}\right\}$ and $\mathcal{B}_{j, 1}(\varphi):=\left\{\sigma_{j+1,2 l+1} \varphi: l=0, \ldots, N_{j}-1\right\}$. For $\mathcal{B}_{j, 0}(\varphi)$, we define a special orthonormality criterion. We say that $\mathcal{B}_{j, 0}(\varphi)$ is orthonormal, if the modified Gramian matrix fulfils

$$
\left(\epsilon_{j, m}\left\langle\sigma_{j, l} \varphi, \sigma_{j, m} \varphi\right\rangle\right)_{1, m=0}^{N_{j}}=\mathbf{I}_{j} .
$$

The system $\mathcal{B}_{j, 1}(\varphi)$ is called orthonormal, if the Gramian matrix satisfies

$$
\left(\left\langle\sigma_{j+1,2 l+1} \varphi, \sigma_{j+1,2 m+1} \varphi\right\rangle\right)_{l, m=0}^{N_{j}-1}=\tilde{\mathbf{I}}_{j} .
$$

Then we obtain the following characterizations for the bases $\mathcal{B}_{j, \nu}(\varphi)(\nu \in\{0,1\})$ in terms of the bracket products.

\section{Lemma 3.5}

Let $\nu \in\{0,1\}$ and $j \in \mathbb{N}_{0}$ be given.

(i) The system $\mathcal{B}_{j, \nu}(\varphi)$ is a basis of $S_{j, \nu}(\varphi)$ if and only if for all $k=0, \ldots, N_{j}-\nu$

$$
[\mathbf{a}[\varphi], \mathbf{a}[\varphi]]_{j, k}>0 \text {. }
$$

(ii) The system $\mathcal{B}_{j, \nu}(\varphi)$ is an orthonormal basis of $S_{j, \nu}(\varphi)$ if and only if

$$
[\mathbf{a}[\varphi], \mathbf{a}[\varphi]]_{j, k}=2 / N_{j} \quad\left(k=0, \ldots, N_{j}-\nu\right) .
$$


(iii) If $\varphi$ satisfies (3.14), and if $\varphi^{*} \in L_{w}^{2}(I)$ is defined by

$$
a_{n}\left[\varphi^{*}\right]:=\hat{c}_{j+1, n}\left(\varphi^{*}\right) a_{n}[\varphi] \quad\left(n \in \mathbb{N}_{0}\right)
$$

with coefficients $\hat{c}_{j+1, n}\left(\varphi^{*}\right)$ determined by (3.7) and

$$
\hat{c}_{j+1, n}\left(\varphi^{*}\right):=\left(2 / N_{j}\right)^{1 / 2}[\mathbf{a}[\varphi], \mathbf{a}[\varphi]]_{j, n}^{-1 / 2} \quad\left(n=0, \ldots, N_{j}-\nu\right),
$$

then $\mathcal{B}_{j, \nu}\left(\varphi^{*}\right)$ is an orthonormal basis of $S_{j, \nu}(\varphi)$.

\section{Proof}

Let $\nu=0$. The system $\mathcal{B}_{j, 0}(\varphi)$ forms a basis of $S_{j, 0}(\varphi)$ if and only if the Gramian matrix

$$
\left(\left\langle\sigma_{j, l} \varphi, \sigma_{j, m} \varphi\right\rangle\right)_{l, m=0}^{N_{j}}
$$

is regular. Since $\mathbf{C}_{j}$ and $\mathbf{D}_{j}$ are regular, by (3.10) this is the case if and only if $\operatorname{diag}[\mathbf{a}[\varphi], \mathbf{a}[\varphi]]_{j}^{\mathrm{I}}$ is regular, i.e., if and only if (3.14) is satisfied.

By definition, $\mathcal{B}_{j, 0}(\varphi)$ is an orthonormal basis of $S_{j, 0}(\varphi)$ if and only if

$$
\left(\epsilon_{j, m}\left\langle\sigma_{j, l} \varphi, \sigma_{j, m} \varphi\right)\right)_{l, m=0}^{N_{j}}=\left(\left\langle\sigma_{j, l} \varphi, \sigma_{j, m} \varphi\right\rangle\right)_{l, m=0}^{N_{j}} \mathbf{D}_{j}=\mathbf{I}_{j}
$$

By (3.10) and (2.6), this is true if and only if (3.15) holds. Finally, by verifying

$$
\left[\mathbf{a}\left[\varphi^{*}\right], \mathbf{a}\left[\varphi^{*}\right]\right]_{j, k}=2 / N_{j} \quad\left(k=0, \ldots, N_{j}\right),
$$

we see that by construction $\mathcal{B}_{j, 0}\left(\varphi^{*}\right)$ is an orthonormal basis of $S_{j, 0}\left(\varphi^{*}\right)$. By lemma 3.2 , the definition of $\varphi^{*}$ implies that $S_{j, 0}\left(\varphi^{*}\right)=S_{j, 0}(\varphi)$.

Using (2.7) and (3.11), the assertions follow analogously for $\nu=1$.

With the help of the bracket product, we are able to give a simple description of the orthogonal projectors $P_{j, \nu}(\nu=0,1)$ of $L_{w}^{2}(I)$ onto $S_{j, \nu}(\varphi)$.

\section{Lemma 3.6}

Let $j \in \mathbb{N}_{0}, \nu \in\{0,1\}$ and let $\varphi \in L_{w}^{2}(I)$ with (3.14) be given. Then for $f \in L_{w}^{2}(I)$ we have

$$
a_{n}\left[P_{j, \nu} f\right]=\hat{c}_{j+1, n}\left(P_{j, \nu} f\right) a_{n}[\varphi] \quad\left(n \in \mathbb{N}_{0}\right),
$$

where the coefficients $\hat{c}_{j+1, n}\left(P_{j, v} f\right)$ satisfy the relations (3.7) and

$$
\hat{c}_{j+1, l}\left(P_{j, \nu} f\right):=\frac{[\mathbf{a}[f], \mathbf{a}[\varphi]]_{j, l}}{[\mathbf{a}[\varphi], \mathbf{a}[\varphi]]_{j, l}} \quad\left(l=0, \ldots, N_{j}-\nu\right) .
$$

The projector $P_{j, \nu}$ is shift-invariant of level $j$, i.e., for all $f \in L_{w}^{2}(I)$ and $k=0, \ldots, N_{j}-\nu$

$$
\sigma_{j, k}\left(P_{j, \nu} f\right)=P_{j, \nu}\left(\sigma_{j, k} f\right)
$$

\section{Proof}

We show the assertion only for $\nu=0$. For $f \in L_{w}^{2}(I)$, the orthogonal projection $P_{j, 0} f \in S_{j, 0}(\varphi)$ is determined by $f-P_{j, 0} f \perp S_{j, 0}(\varphi)$. Then there are coefficients 
$\hat{c}_{j+1, n}\left(P_{j, 0} f\right)\left(n \in \mathbb{N}_{0}\right)$ satisfying the properties (3.7) of symmetry and periodicity with

$$
a_{n}\left[P_{j, 0} f\right]=\hat{c}_{j+1, n}\left(P_{j, 0} f\right) a_{n}[\varphi] \quad\left(n \in \mathbb{N}_{0}\right) .
$$

Using lemma 3.3, we obtain for all $l=0, \ldots, N_{j}$

$$
\begin{aligned}
0 & =\left\langle f-P_{j, 0} f, \sigma_{j, l} \varphi\right\rangle \\
& =\sum_{k=0}^{N_{j}} \epsilon_{j, k} \cos \frac{k l \pi}{N_{j}}\left[\mathbf{a}\left[f-P_{j, 0} f\right], \mathbf{a}[\varphi]\right]_{j, k} \\
& =\sum_{k=0}^{N_{j}} \epsilon_{j, k} \cos \frac{k l \pi}{N_{j}}\left([\mathbf{a}[f], \mathbf{a}[\varphi]]_{j, k}-\hat{c}_{j+1, k}\left(P_{j, 0} f\right)[\mathbf{a}[\varphi], \mathbf{a}[\varphi]]_{j, k}\right) .
\end{aligned}
$$

Hence the coefficients $\hat{c}_{j+1, k}\left(P_{j, 0} f\right)$ satisfy (3.16). The shift-invariance of $P_{j, 0}$ follows from

$$
\begin{aligned}
a_{n}\left[\sigma_{j, l}\left(P_{j, 0} f\right)\right] & =\hat{c}_{j+1, n}\left(P_{j, 0} f\right) a_{n}[\varphi] \cos \frac{\ln \pi}{N_{j}} \\
& =\hat{c}_{j+1, n}\left(P_{j, 0}\left(\sigma_{j, l} f\right)\right) a_{n}[\varphi]=a_{n}\left[P_{j, 0}\left(\sigma_{j, l} f\right)\right] \quad\left(n \in \mathbb{N}_{0}\right) .
\end{aligned}
$$

\section{Multiresolution of $L_{w}^{2}(I)$}

We form shift-invariant subspaces $V_{j}:=S_{j, 0}\left(\varphi_{j}\right)$ with $\varphi_{j} \in L_{w}^{2}(I)$ for each level $j \in \mathbb{N}_{0}$. The sequence of subspaces $V_{j}\left(j \in \mathbb{N}_{0}\right)$ is called a nonstationary multiresolution of $L_{w}^{2}(I)$, if the following three conditions are satisfied:

(M1) $V_{j} \subset V_{j+1}\left(j \in \mathbb{N}_{0}\right)$.

(M2) $\operatorname{clos}\left(\bigcup_{j=0}^{\infty} V_{j}\right)=L_{w}^{2}(I)$.

(M3) The systems $\mathcal{B}_{j, 0}\left(\left(N_{j} / 2\right)^{1 / 2} \varphi_{j}\right)\left(j \in \mathbb{N}_{0}\right)$ are $L_{w}^{2}(I)$-stable, i.e., there exist positive constants $\alpha, \beta$ independent of $j$ such that for all $j \in \mathbb{N}_{0}$ and for any $\left(\alpha_{j, n}\right)_{n=0}^{N_{j}} \in \mathbb{R}^{N_{j}+1}$,

$$
\alpha \sum_{n=0}^{N_{j}} \epsilon_{j, n} \alpha_{j, n}^{2} \leq\left\|\sum_{n=0}^{N_{j}} \epsilon_{j, n} \alpha_{j, n}\left(N_{j} / 2\right)^{1 / 2} \sigma_{j, n} \varphi_{j}\right\|^{2} \leq \beta \sum_{n=0}^{N_{j}} \epsilon_{j, n} \alpha_{j, n}^{2} .
$$

By $(\mathrm{M} 3), \mathcal{B}_{j, 0}\left(\left(N_{j} / 2\right)^{1 / 2} \varphi_{j}\right)$ is a basis of $V_{j}$. Note that $\operatorname{dim} V_{j}=N_{j}+1$. The shiftinvariant subspace $V_{j}$ is called sample space of level $j$. The function $\varphi_{j}$ of $V_{j}$ is said to be the scaling function of $V_{j}$. If all systems $\mathcal{B}_{j, 0}\left(\left(N_{j} / 2\right)^{1 / 2} \varphi_{j}\right)$ are orthonormal bases of $V_{j}\left(j \in \mathbb{N}_{0}\right)$ in the sense of (3.13), then we say that $\left(N_{j} / 2\right)^{1 / 2} \varphi_{j}\left(j \in \mathbb{N}_{0}\right)$ are orthonormal scaling functions. In this case the constants in condition (M3) are $\alpha=\beta=1$. Concerning (M2), we observe the following 


\section{Theorem 4.1}

Let $\left\{V_{j}\right\}_{j=0}^{\infty}$ be a nested sequence of shift-invariant subspaces $V_{j}:=S_{j, 0}\left(\varphi_{j}\right)$ with $\varphi_{j} \in L_{w}^{2}(I)$, i.e., (M1) is valid. Then the condition (M2) is satisfied if and only if

$$
\bigcup_{j=0}^{\infty} \operatorname{supp} \mathbf{a}\left[\varphi_{j}\right]=\mathbb{N}_{0} \text {. }
$$

Proof

1) Suppose that (4.2) is not satisfied. Then there is a number

$$
n_{0} \in \mathbb{N}_{0} \backslash \bigcup_{j=0}^{\infty} \operatorname{supp} \mathbf{a}\left[\varphi_{j}\right]
$$

such that for the Chebyshev polynomial $T_{n_{0}}$ it holds that

$$
T_{n_{0}} \perp \operatorname{clos}\left(\bigcup_{j=0}^{\infty} V_{j}\right) \text {. }
$$

Thus, (M2) is not satisfied.

2) Assume that (4.2) holds. By (M1) and lemma 3.2, we have

$$
\operatorname{supp} \mathbf{a}\left[\varphi_{j}\right] \subseteq \operatorname{supp} \mathbf{a}\left[\varphi_{j+1}\right] \quad\left(j \in \mathbb{N}_{0}\right) \text {. }
$$

Suppose that there exists $f \in L_{w}^{2}(I)(f \neq 0)$ with

$$
f \perp \operatorname{clos}\left(\bigcup_{j=0}^{\infty} V_{j}\right) \text {. }
$$

By $k_{0} \in \mathbb{N}_{0}$, we denote an index for which

$$
\left|a_{k_{0}}[f]\right|=\max \left\{\left|a_{k}[f]\right|: k \in \mathbb{N}_{0}\right\}>0 .
$$

By (4.2)-(4.3) we conclude that there is an index $j_{0} \in \mathbb{N}_{0}$ such that $k_{0} \in \operatorname{supp} \mathbf{a}\left[\varphi_{j_{0}}\right]$ and $N_{j_{0}} \geq k_{0}$. Since $\varphi_{j_{0}} \in V_{j}$ for all $j \geq j_{0}$, we find that $f \perp S_{j, 0}\left(\varphi_{j_{0}}\right)\left(j \geq j_{0}\right)$. Hence, for $j \geq j_{0}$, we have by (3.12)

$$
\left[\mathbf{a}[f], \mathbf{a}\left[\varphi_{j_{0}}\right]\right]_{j, k_{0}}=0,
$$

i.e.,

$$
\begin{aligned}
& a_{k_{0}}[f] a_{k_{0}}\left[\varphi_{j_{0}}\right]+a_{N_{j+1}-k_{0}}[f] a_{N_{j+1}-k_{0}}\left[\varphi_{j_{0}}\right] \\
& \quad+\sum_{n=1}^{\infty}\left(a_{k_{0}+n N_{j+1}}[f] a_{k_{0}+n N_{j+1}}\left[\varphi_{j_{0}}\right]+a_{(n+1) N_{j+1}-k_{0}}[f] a_{(n+1) N_{j+1}-k_{0}}\left[\varphi_{j_{0}}\right]\right)=0 .
\end{aligned}
$$

Put

$$
\epsilon_{0}:=\left|a_{k_{0}}[f] a_{k_{0}}\left[\varphi_{j_{0}}\right]\right|>0,
$$


and choose $j_{\mathrm{l}} \geq j_{0}$ such that

$$
\sum_{n \geq N_{j_{1}}}\left|a_{n}[f] a_{n}\left[\varphi_{j_{0}}\right]\right| \leq \epsilon_{0} / 2
$$

This choice of $j_{1}$ is possible, since by the Cauchy-Schwarz inequality

$$
\sum_{n=1}^{\infty}\left|a_{n}[f] a_{n}\left[\varphi_{j_{0}}\right]\right| \leq\|\mathbf{a}[f]\|_{1^{2}}\left\|\mathbf{a}\left[\varphi_{j_{0}}\right]\right\|_{I^{2}}<\infty .
$$

But (4.6) contradicts equation (4.5) for $j=j_{1}$. This implies that $f=0$, i.e., (M2) is satisfied.

\section{Theorem 4.2}

The system $\left\{\mathcal{B}_{j, 0}\left(\left(N_{j} / 2\right)^{1 / 2} \varphi_{j}\right): j \in \mathbb{N}_{0}\right\}$ is $L_{w}^{2}(I)$-stable with positive constants $\alpha, \beta$ independent of $j$ if and only if for all $k=0, \ldots, N_{j}$ and for all $j \in \mathbb{N}_{0}$

$$
\alpha \leq \frac{N_{j}^{2}}{4}\left[\mathbf{a}\left[\varphi_{j}\right], \mathbf{a}\left[\varphi_{j}\right]\right]_{j, k} \leq \beta
$$

\section{Proof}

1) From lemma 3.3 , it follows that for $j \in \mathbb{N}_{0}$ and $\left(\alpha_{j, k}\right)_{k=0}^{N_{j}} \in \mathbb{R}^{N_{j}+1}$

$$
\left\|\sum_{k=0}^{N_{j}} \epsilon_{j, k} \alpha_{j, k}\left(N_{j} / 2\right)^{1 / 2} \sigma_{j, k} \varphi_{j}\right\|^{2}=\frac{N_{j}}{2} \sum_{n=0}^{N_{j}} \epsilon_{j, n} \hat{\alpha}_{j, n}^{2}\left[\mathbf{a}\left[\varphi_{j}\right], \mathbf{a}\left[\varphi_{j}\right]\right]_{j, n}
$$

with

$$
\hat{\alpha}_{j, n}:=\sum_{k=0}^{N_{j}} \epsilon_{j, k} \alpha_{j, k} \cos \frac{k n \pi}{N_{j}} \quad\left(n \in \mathbb{N}_{0}\right) .
$$

By (2.10)-(2.11) we have

$$
\sum_{k=0}^{N_{j}} \epsilon_{j, k} \alpha_{j, k}^{2}=\frac{2}{N_{j}} \sum_{n=0}^{N_{j}} \epsilon_{j, n} \hat{\alpha}_{j, n}^{2} .
$$

With the considerations above, (4.1) reads as follows

$$
\alpha \sum_{n=0}^{N_{j}} \epsilon_{j, n} \hat{\alpha}_{j, n}^{2} \leq \frac{N_{j}^{2}}{4} \sum_{n=0}^{N_{j}} \epsilon_{j, n} \hat{\alpha}_{j, n}^{2}\left[\mathbf{a}\left[\varphi_{j}\right], \mathbf{a}\left[\varphi_{j}\right]\right]_{j, n} \leq \beta \sum_{n=0}^{N_{j}} \epsilon_{j, n} \hat{\alpha}_{j, n}^{2},
$$

with arbitrary $\left(\hat{\alpha}_{j, n}\right)_{n=0}^{N_{j}} \in \mathbb{R}^{N_{j}+1}$ and $j \in \mathbb{N}_{0}$, which is equivalent to (4.7).

In the following, we assume that (M1)-(M3) are satisfied. From (M1) it follows $\varphi_{j} \in V_{j+1}$, i.e., there exist unique coefficients $\alpha_{j+1, k}\left(\varphi_{j}\right) \in \mathbb{R}\left(k=0, \ldots, N_{j+1}\right)$ such that

$$
\varphi_{j}=\sum_{k=0}^{N_{j+1}} \epsilon_{j+1, k} \alpha_{j+1, k}\left(\varphi_{j}\right) \sigma_{j+1, k} \varphi_{j+1}
$$


This is the so-called two-scale relation or refinement equation of $\varphi_{j}$. The Chebyshev transformed two-scale relation of $\varphi_{j}$ reads

$$
a_{n}\left[\varphi_{j}\right]=A_{j+1}(n) a_{n}\left[\varphi_{j+1}\right] \quad\left(n \in \mathbb{N}_{0}\right)
$$

with the two-scale symbol or refinement mask of $\varphi_{j}$

$$
A_{j+1}(n):=\sum_{k=0}^{N_{j+1}} \epsilon_{j+1, k} \alpha_{j+1, k}\left(\varphi_{j}\right) \cos \frac{k n \pi}{N_{j+1}} \quad\left(n \in \mathbb{N}_{0}\right) .
$$

By definition we obtain the relations of periodicity and symmetry for all $n \in \mathbb{N}_{0}$ and $l=0, \ldots, N_{j+2}-1$,

$$
A_{j+1}(n)=A_{j+1}\left(N_{j+2}+n\right), \quad A_{j+1}\left(N_{j+2}-l\right)=A_{j+1}(l) .
$$

If a scaling function $\varphi_{j}\left(j \in \mathbb{N}_{0}\right)$ satisfying (4.7) is given, then an orthonormal basis $\mathcal{B}_{j, 0}\left(\left(N_{j} / 2\right)^{1 / 2} \varphi_{j}^{*}\right)\left(j \in \mathbb{N}_{0}\right)$ can be easily obtained by lemma 3.5 , (iii). Let $\varphi_{j}^{*}\left(j \in \mathbb{N}_{0}\right)$ be defined by its Chebyshev coefficients

$$
\frac{N_{j}}{2} a_{n}\left[\varphi_{j}^{*}\right]:=\left(\left[\mathbf{a}\left[\varphi_{j}\right], \mathbf{a}\left[\varphi_{j}\right]\right]_{j, n}\right)^{-1 / 2} a_{n}\left[\varphi_{j}\right] \quad\left(n \in \mathbb{N}_{0}\right)
$$

Then $\mathcal{B}_{j, 0}\left(\left(N_{j} / 2\right)^{1 / 2} \varphi_{j}^{*}\right)$ is an orthonormal basis of $V_{j}=S_{j, 0}\left(\varphi_{j}\right)$. The two-scale symbol $A_{j+1}^{*}$ satisfying

$$
a_{n}\left[\varphi_{j}^{*}\right]=A_{j+1}^{*}(n) a_{n}\left[\varphi_{j+1}^{*}\right] \quad\left(n \in \mathbb{N}_{0}\right)
$$

is connected with $A_{j+1}$ by

$$
A_{j+1}^{*}(n):=2\left(\frac{\left[\mathbf{a}\left[\varphi_{j+1}\right], \mathbf{a}\left[\varphi_{j+1}\right]\right]_{j+1, n}}{\left[\mathbf{a}\left[\varphi_{j}\right], \mathbf{a}\left[\varphi_{j}\right]\right]_{j, n}}\right)^{1 / 2} A_{j+1}(n) \quad\left(n \in \mathbb{N}_{0}\right) .
$$

The following connection between the bracket product $\left[\mathbf{a}\left[\varphi_{j}\right], \mathbf{a}\left[\varphi_{j}\right]\right]_{j}^{1}$ and the two-scale symbol $A_{j+1}$ can be observed:

\section{Lemma 4.3}

For $j \in \mathbb{N}_{0}$ and $k=0, \ldots, N_{j}$, we have

$$
\begin{aligned}
{\left[\mathbf{a}\left[\varphi_{j}\right], \mathbf{a}\left[\varphi_{j}\right]\right]_{j, k}=} & A_{j+1}(k)^{2}\left[\mathbf{a}\left[\varphi_{j+1}\right], \mathbf{a}\left[\varphi_{j+1}\right]\right]_{j+1, k} \\
& +A_{j+1}\left(N_{j+1}-k\right)^{2}\left[\mathbf{a}\left[\varphi_{j+1}\right], \mathbf{a}\left[\varphi_{j+1}\right]\right]_{j+1, N_{j+1}-k}
\end{aligned}
$$

In particular, if $\left(N_{j} / 2\right)^{1 / 2} \varphi_{j}^{*}$ is an orthonormal scaling function and if $A_{j+1}^{*}$ is the two-scale symbol of $\varphi_{j}^{*}$, then

$$
A_{j+1}^{*}(k)^{2}+A_{j+1}^{*}\left(N_{j+1}-k\right)^{2}=4 \quad\left(k=0, \ldots, N_{j}\right) .
$$




\section{Proof}

By the definition of the bracket product and by (4.8)-(4.9), we obtain for $k=0, \ldots, N_{j}$

$$
\begin{aligned}
{\left[\mathbf{a}\left[\varphi_{j}\right]\right.} & \left., \mathbf{a}\left[\varphi_{j}\right]\right]_{j, k} \\
& =\sum_{n=0}^{\infty}\left(a_{n N_{j+2}+k}\left[\varphi_{j}\right]^{2}+a_{(n+1) N_{j+2}-k}\left[\varphi_{j}\right]^{2}+a_{n N_{j+2}+N_{j+1}+k}\left[\varphi_{j}\right]^{2}+a_{n N_{j+2}+N_{j+1}-k}\left[\varphi_{j}\right]^{2}\right) \\
& =A_{j+1}(k)^{2}\left[\mathbf{a}\left[\varphi_{j+1}\right], \mathbf{a}\left[\varphi_{j+1}\right]\right]_{j+1, k}+A_{j+1}\left(N_{j+1}-k\right)^{2}\left[\mathbf{a}\left[\varphi_{j+1}\right], \mathbf{a}\left[\varphi_{j+1}\right]\right]_{j+1, N_{j+1}-k} .
\end{aligned}
$$

For orthonormal scaling functions, the assertion follows by lemma 3.5, (ii).

\section{Wavelet spaces}

Let the wavelet space $W_{j}$ of level $j\left(j \in \mathbb{N}_{0}\right)$ be defined as the orthogonal complement of $V_{j}$ in $V_{j+1}$, i.e.

$$
W_{j}:=V_{i+1} \ominus V_{j} \quad\left(j \in \mathbb{N}_{0}\right) .
$$

Then it follows that $\operatorname{dim} W_{j}=\left(N_{j+1}+1\right)-\left(N_{j}+1\right)=N_{j}$. By definition, the wavelet spaces $W_{j}\left(j \in \mathbb{N}_{0}\right)$ are orthogonal. By (M1)-(M2), we obtain the orthogonal sum decomposition

$$
L_{w}^{2}(I)=V_{0} \oplus \bigoplus_{j=0}^{\infty} W_{j}
$$

Further, $W_{j}$ can be characterized by the orthogonal projector $P_{j, 0}$ of $L_{w}^{2}(I)$ onto $V_{j}$, namely by

$$
W_{j}=\left\{f-P_{j, 0} f: f \in V_{j+1}\right\} .
$$

The subspace $W_{j}$ is shift-invariant of level $j$, since by lemma 3.6 we have for $g:=f-P_{j, 0} f\left(f \in V_{j+1}\right)$,

$$
\sigma_{j, l} g=\sigma_{j, l} f-\sigma_{j, l}\left(P_{j, 0} f\right)=\sigma_{j, l} f-P_{j, 0}\left(\sigma_{j, l} f\right) \in W_{j} .
$$

Assume that the shift-invariant subspace $W_{j}$ can be of type 1 generated by a function $\psi_{j} \in V_{j+1}$ such that $W_{j}=S_{j, 1}\left(\psi_{j}\right)$. Further, we suppose that the set $\mathcal{B}_{j, 1}\left(\left(N_{j} / 2\right)^{1 / 2} \psi_{j}\right)=\left\{\left(N_{j} / 2\right)^{1 / 2} \sigma_{j+1,2 l+1} \psi_{j}: l=0, \ldots, N_{j}-1\right\}$ is $L_{w}^{2}(I)$-stable, i.e., there are constants $0<\gamma \leq \delta<\infty$ independent of $j$ such that for all $j \in \mathbb{N}_{0}$ and for any $\left(\beta_{j, n}\right)_{n=0}^{N_{j}-1} \in \mathbb{R}^{N_{j}}$,

$$
\gamma \sum_{n=0}^{N_{j}-1} \beta_{j, n}^{2} \leq\left\|\sum_{n=0}^{N_{j}-1} \beta_{j, n}\left(N_{j} / 2\right)^{1 / 2} \sigma_{j+1,2 n+1} \psi_{j}\right\|^{2} \leq \delta \sum_{n=0}^{N_{j}-1} \beta_{j, n}^{2} .
$$

Under these assumptions, $\psi_{j}$ is called semiorthogonal wavelet. If $\mathcal{B}_{j, 1}\left(\left(N_{j} / 2\right)^{1 / 2} \psi_{j}\right)$ $\left(j \in \mathbb{N}_{0}\right)$ are orthonormal bases, then $\left(N_{j} / 2\right)^{1 / 2} \psi_{j}$ are called orthonormal wavelets. Obviously, for orthonormal wavelets the condition (5.1) is satisfied with $\gamma=\delta=1$. 
By $W_{j} \subset V_{j+1}$, there are unique coefficients $\alpha_{j+1, k}\left(\psi_{j}\right) \in \mathbb{R}\left(k=0, \ldots, N_{j+1}\right)$ such that a two-scale relation or refinement equation of $\psi_{j}$ of the form

$$
\psi_{j}=\sum_{k=0}^{N_{j+1}} \epsilon_{j+1, k} \alpha_{j+1, k}\left(\psi_{j}\right) \sigma_{j+1, k} \phi_{j+1}
$$

is satisfied. By means of the Chebyshev transform this yields

$$
\begin{aligned}
a_{n}\left[\psi_{j}\right] & =B_{j+1}(n) a_{n}\left[\varphi_{j+1}\right] & & \left(n \in \mathbb{N}_{0}\right), \\
a_{n}\left[\sigma_{j+1,1} \psi_{j}\right] & =\cos \frac{n \pi}{N_{j+1}} B_{j+1}(n) a_{n}\left[\varphi_{j+1}\right] & & \left(n \in \mathbb{N}_{0}\right)
\end{aligned}
$$

with the two-scale symbol or refinement mask of $\psi_{j}$

$$
B_{j+1}(n):=\sum_{k=0}^{N_{j+1}} \epsilon_{j+1, k} \alpha_{j+1, k}\left(\psi_{j}\right) \cos \frac{k n \pi}{N_{j+1}} \quad\left(n \in \mathbb{N}_{0}\right) .
$$

It is clear that $B_{j+1}$ satisfies the same properties of periodicity and symmetry as $A_{j+1}$ in (4.9). As in the sample space $V_{j}$, the bracket products are important for the characterization of the $L_{w}^{2}(I)$-stability of $W_{j}$ and the orthogonality $W_{j} \perp V_{j}$ :

\section{Theorem 5.1}

(i) The condition (5.1) for $j \in \mathbb{N}_{0}$ with positive constants $\gamma$ and $\delta$ independent of $j$ is equivalent to

$$
\gamma \leq \frac{N_{j}^{2}}{4}\left[\mathbf{a}\left[\psi_{j}\right], \mathbf{a}\left[\psi_{j}\right]\right]_{j, n} \leq \delta \quad\left(n=0, \ldots, N_{j}-1\right)
$$

(ii) For $j \in \mathbb{N}_{0}$ and $k=0, \ldots, N_{j}-1$ we have

$$
\begin{gathered}
{\left[\mathbf{a}\left[\sigma_{j+1,1} \psi_{j}\right], \mathbf{a}\left[\sigma_{j+1,1} \psi_{j}\right]\right]_{j, k}=\left(\cos \frac{k \pi}{N_{j+1}}\right)^{2}\left[\mathbf{a}\left[\psi_{j}\right], \mathbf{a}\left[\psi_{j}\right]\right]_{j, k}} \\
=\left(\cos \frac{k \pi}{N_{j+1}}\right)^{2}\left(B_{j+1}(k)^{2}\left[\mathbf{a}\left[\varphi_{j+1}\right], \mathbf{a}\left[\varphi_{j+1}\right]\right]_{j+1, k}\right. \\
\left.+B_{j+1}\left(N_{j+1}-k\right)^{2}\left[\mathbf{a}\left[\varphi_{j+1}\right], \mathbf{a}\left[\varphi_{j+1}\right]\right]_{j+1, N_{j+1}-k}\right) .
\end{gathered}
$$

(iii) For $j \in \mathbb{N}_{0}$, we have $S_{j, 1}\left(\psi_{j}\right) \perp V_{j}$ if and only if for all $n=0, \ldots, N_{j}-1$

$$
\begin{aligned}
& A_{j+1}(n) B_{j+1}(n)\left[\mathbf{a}\left[\varphi_{j+1}\right], \mathbf{a}\left[\varphi_{j+1}\right]\right]_{j+1, n} \\
& \quad-A_{j+1}\left(N_{j+1}-n\right) B_{j+1}\left(N_{j+1}-n\right)\left[\mathbf{a}\left[\varphi_{j+1}\right], \mathbf{a}\left[\varphi_{j+1}\right]\right]_{j+1, N_{j+1}-n}=0 .
\end{aligned}
$$

Proof

The proofs for (i) and (ii) are similar to those of theorem 4.2 and lemma 4.3. In order to show (iii), we observe that $V_{j} \perp S_{j, 1}\left(\psi_{j}\right)=S_{j, 0}\left(\sigma_{j+1,1} \psi_{j}\right)$ is equivalent to the equations $\left[\mathbf{a}\left[\varphi_{j}\right], \mathbf{a}\left[\sigma_{j+1,1} \psi_{j}\right]\right]_{j, k}=0$ for all $k=0, \ldots, N_{j}$ by corollary 3.4 , (i). Inserting the two-scale relations (4.8) and (5.2), we obtain the assertion. 
Note that from (5.5) it follows that this equation is also valid for $n=$ $N_{j}+1, \ldots, N_{j+1}$.

We introduce the two-scale symbol matrices of level $j\left(j \in \mathbb{N}_{0}\right)$ for $n=0, \ldots, N_{j}-1$ by

$$
\mathbf{S}_{j+1}(n):=\left(\begin{array}{cc}
A_{j+1}(n) & B_{j+1}(n) \\
A_{j+1}\left(N_{j+1}-n\right) & -B_{j+1}\left(N_{j+1}-n\right)
\end{array}\right) .
$$

As usual, these matrices will play an important role in deriving the decomposition and reconstruction algorithms. Therefore we have to investigate the invertibility of $\mathbf{S}_{j+1}(n)$. Let $\lambda_{\nu}(\nu=0,1)$ be the eigenvalues of $\mathbf{S}_{j+1}(n)$, i.e., it holds that $\operatorname{det}\left(\mathbf{S}_{j+1}(n)-\lambda_{\nu} \mathbf{I}\right)=0$ with the unit matrix $\mathbf{I}$.

\section{Lemma 5.2}

Assume that (M1)-(M3) and (5.1) hold with positive constants $\alpha, \beta, \gamma, \delta$. Then the two-scale symbol matrices $\mathbf{S}_{j+1}(n)$ are regular for all $n=0, \ldots, N_{j}-1$ satisfying

$$
\frac{4}{\beta} \min \{\alpha, \gamma\} \leq\left|\lambda_{\nu}\right|^{2} \leq \frac{4}{\alpha} \max \{\beta, \delta\} \quad(\nu=0,1) .
$$

In particular, it holds that

$$
\frac{4}{\beta} \sqrt{\alpha \gamma} \leq\left|\operatorname{det} \mathbf{S}_{j+1}(n)\right| \leq \frac{4}{\alpha} \sqrt{\beta \delta} \quad\left(n=0, \ldots, N_{j}-1\right) .
$$

Furthermore, we have for $n=0, \ldots, N_{j}-1$

$$
\begin{aligned}
\mathbf{S}_{j+1}(n)^{-1}= & \operatorname{diag}\left(\left[\mathbf{a}\left[\varphi_{j}\right], \mathbf{a}\left[\varphi_{j}\right]\right]_{j, n}^{-1},\left[\mathbf{a}\left[\psi_{j}\right], \mathbf{a}\left[\psi_{j}\right]_{j, n}^{-1}\right)^{\mathrm{T}} \mathbf{S}_{j+1}(n)^{\mathrm{T}}\right. \\
& \times \operatorname{diag}\left(\left[\mathbf{a}\left[\varphi_{j+1}\right], \mathbf{a}\left[\varphi_{j+1}\right]\right]_{j+1, n},\left[\mathbf{a}\left[\varphi_{j+1}\right], \mathbf{a}\left[\varphi_{j+1}\right]\right]_{j+1, N_{j+1}-n}\right)^{\mathrm{T}} .
\end{aligned}
$$

\section{Proof}

Using lemma 4.3 and (5.4)-(5.5), we find for $n=0, \ldots, N_{j}-1$

$$
\begin{gathered}
\mathbf{S}_{j+1}(n)^{\mathrm{T}} \operatorname{diag}\left(\left[\mathbf{a}\left[\varphi_{j+1}\right], \mathbf{a}\left[\varphi_{j+1} \mid\right]\right]_{j+1, n},\left[\mathbf{a}\left[\varphi_{j+1}\right], \mathbf{a}\left[\varphi_{j+1}\right]\right]_{j+1, N_{j+1}-n}\right)^{\mathrm{T}} \mathbf{S}_{j+1}(n) \\
=\operatorname{diag}\left(\left[\mathbf{a}\left[\varphi_{j}\right], \mathbf{a}\left[\varphi_{j}\right]\right]_{j, n},\left[\mathbf{a}\left[\psi_{j}\right], \mathbf{a}\left[\psi_{j}\right]\right]_{j, n}\right)^{\mathrm{T}} .
\end{gathered}
$$

Thus (5.9) holds for $n=0, \ldots, N_{j}-1$. By (5.9), (4.7) and (5.3), the eigenvalues and the determinant of $\mathbf{S}_{j+1}(n)$ can be easily estimated in terms of the constants $\alpha, \beta$, $\gamma, \delta$.

Now by the help of the conditions for the two-scale symbol $B_{j+1}$ of $\psi_{j}$ in theorem 5.1, we obtain

\section{Theorem 5.3}

Assume that (M1)-(M3) are fulfilled. Then for all $j \in \mathbb{N}_{0}, B_{j+1}: \mathbb{N}_{0} \rightarrow \mathbb{R}$ is a two-scale symbol of a semiorthogonal wavelet $\psi_{j} \in L_{w}^{2}(I)$ if and only if for 
$n=0, \ldots, N_{j+1}$, the two-scale symbol $B_{j+1}(n)$ is of the form

$$
B_{j+1}(n)=\frac{\left[\mathbf{a}\left[\varphi_{j+1}\right], \mathbf{a}\left[\varphi_{j+1}\right]\right]_{j+1, N_{j+1}-n} A_{j+1}\left(N_{j+1}-n\right)}{\left[\mathbf{a}\left[\varphi_{j}\right], \mathbf{a}\left[\varphi_{j}\right]\right]_{j, n}} K_{j}(n),
$$

where $B_{j+1}$ has the same properties (4.9) of periodicity and symmetry as $A_{j+1}$, and where $K_{j}: \mathbb{N}_{0} \rightarrow \mathbb{R}$ satisfies the conditions

$$
\begin{aligned}
0<\nu \leq\left|K_{j}(n)\right| & \leq \mu<\infty & & \left(n=0, \ldots, N_{j}-1\right) \\
K_{j}(n) & =K_{j}\left(n+N_{j+1}\right) & & \left(n \in \mathbb{N}_{0}\right), \\
K_{j}\left(N_{j+1}-n\right) & =K_{j}(n) & & \left(n=0, \ldots, N_{j}-1\right)
\end{aligned}
$$

for some constants $\nu$ and $\mu$.

\section{Proof}

1) Let $B_{j+1}$ be given in the form (5.10) with $K_{j}$ satisfying (5.11). Then by theorem 5.1, (iii) the orthogonality $V_{j} \perp S_{j, 1}\left(\psi_{j}\right)$ is satisfied, since for $n=0, \ldots, N_{j}-1$,

$$
\begin{aligned}
& A_{j+1}(n) B_{j+1}(n)\left[\mathbf{a}\left[\varphi_{j+1}\right], \mathbf{a}\left[\varphi_{j+1}\right]\right]_{j+1, n} \\
& \quad-A_{j+1}\left(N_{j+1}-n\right) B_{j+1}\left(N_{j+1}-n\right)\left[\mathbf{a}\left[\varphi_{j+1}\right], \mathbf{a}\left[\varphi_{j+1}\right]\right]_{j+1, N_{j+1}-n}=0 .
\end{aligned}
$$

It follows that $S_{j, 1}\left(\psi_{j}\right) \subseteq W_{j}$. It remains to show that $\mathcal{B}_{j, 1}\left(\left(N_{j} / 2\right)^{1 / 2} \psi_{j}\right)\left(j \in \mathbb{N}_{0}\right)$ are $L_{w}^{2}(I)$-stable. By (5.4) and lemma 4.3, we find for $n=0, \ldots, N_{j}-1$

$$
\left[\mathbf{a}\left[\psi_{j}\right], \mathbf{a}\left[\psi_{j}\right]\right]_{j, n}=\frac{\left[\mathbf{a}\left[\varphi_{j+1}\right], \mathbf{a}\left[\varphi_{j+1}\right]\right]_{j+1, n}\left[\mathbf{a}\left[\varphi_{j+1}\right], \mathbf{a}\left[\varphi_{j+1}\right]\right]_{j+1, N_{j+1}-n}}{\left[\mathbf{a}\left[\varphi_{j}\right], \mathbf{a}\left[\varphi_{j}\right]\right]_{j, n}} K_{j}(n)^{2} .
$$

By (4.7) and (5.11), we can estimate

$$
0<\frac{\alpha^{2}}{16 \beta} \nu^{2} \leq \frac{N_{j}^{2}}{4}\left[\mathbf{a}\left[\psi_{j}\right], \mathbf{a}\left[\psi_{j}\right]\right]_{j, n} \leq \frac{\beta^{2}}{16 \alpha} \mu^{2}<\infty
$$

2) For each $j \in \mathbb{N}_{0}$, let $B_{j+1}: \mathbb{N}_{0} \rightarrow \mathbb{R}$ be the two-scale symbol of a semiorthogonal wavelet $\psi_{j}$ and let $\mathcal{B}_{j, 1}\left(\left(N_{j} / 2\right)^{1 / 2} \psi_{j}\right)\left(j \in \mathbb{N}_{0}\right)$ be $L_{w}^{2}(I)$-stable. Thus $B_{j+1}$ satisfies (5.4) and (5.5). Now, put for $n=0, \ldots, N_{j+1}$

$$
K_{j}(n):=A_{j+1}(n) B_{j+1}\left(N_{j+1}-n\right)+A_{j+1}\left(N_{j+1}-n\right) B_{j+1}(n) .
$$

Note that $K_{j}(n)=K_{j}\left(N_{j+1}-n\right)$ for $n=0, \ldots, N_{j}-1$. Then we continue $K_{j}$ on $\mathbb{N}_{0}$ by $K_{j}\left(n+r N_{j+1}\right):=K_{j}(n)$ for all $n=0, \ldots, N_{j+1}-1$ and $r \in \mathbb{N}_{0}$. Thus $K_{j}$ satisfies the conditions (5.11). Multiplying (5.5) with $A_{j+1}(n)$, by lemma 4.3 we obtain for $n=0, \ldots, N_{j}-1$ and also for $n=N_{j}+1, \ldots, N_{j+1}$

$$
\begin{aligned}
0= & A_{j+1}(n)^{2} B_{j+1}(n)\left[\mathbf{a}\left[\varphi_{j+1}\right], \mathbf{a}\left[\varphi_{j+1}\right]\right]_{j+1, n} \\
& -A_{j+1}(n) A_{j+1}\left(N_{j+1}-n\right) B_{j+1}\left(N_{j+1}-n\right)\left[\mathbf{a}\left[\varphi_{j+1}\right], \mathbf{a}\left[\varphi_{j+1}\right]\right]_{j+1, N_{j+1}-n} \\
= & B_{j+1}(n)\left[\mathbf{a}\left[\varphi_{j}\right], \mathbf{a}\left[\varphi_{j}\right]\right]_{j, n}-K_{j}(n) A_{j+1}\left(N_{j+1}-n\right)\left[\mathbf{a}\left[\varphi_{j+1}\right], \mathbf{a}\left[\varphi_{j+1} 1\right]_{j+1, N_{j+1}-n} .\right.
\end{aligned}
$$

Hence, $B_{j+1}(n)$ is of the form (5.10) for $n=0, \ldots, N_{j}-1, N_{j}+1, \ldots, N_{j+1}$. Defining $B_{j+1}\left(N_{j}\right)$ by $(5.10)$ for $n=N_{j}$, the proof is complete. 


\section{Corollary 5.4}

Assume that $\mathcal{B}_{j, 0}\left(\left(N_{j} / 2\right)^{1 / 2} \varphi_{j}^{*}\right)\left(j \in \mathbb{N}_{0}\right)$ are orthonormal bases of $V_{j}$. Let $A_{j+1}^{*}$ be the two-scale symbols of $\varphi_{j}^{*} \in L_{w}^{2}(I)$. Then for every $j \in \mathbb{N}_{0}, B_{j+1}^{*}: \mathbb{N}_{0} \rightarrow \mathbb{R}$ is a two-scale symbol of a wavelet $\psi_{j}^{*} \in L_{w}^{2}(I)$ generating an orthonormal basis $\mathcal{B}_{j, 1}\left(\left(N_{j} / 2\right)^{1 / 2} \psi_{j}^{*}\right)$ of $W_{j}$ if and only if $B_{j+1}^{*}$ possesses the form

$$
B_{j+1}^{*}(n)= \pm A_{j+1}^{*}\left(N_{j+1}-n\right) \quad\left(n=0, \ldots, N_{j+1}\right)
$$

and fulfils the same properties (4.9) of periodicity and symmetry as $A_{j+1}$.

Proof

By lemma 3.5, (ii) we have

$$
\begin{array}{ll}
N_{j}^{2}\left[\mathbf{a}\left[\varphi_{j}^{*}\right], \mathbf{a}\left[\varphi_{j}^{*}\right]\right]_{j, n}=4 & \left(n=0, \ldots, N_{j}\right), \\
N_{j}^{2}\left[\mathbf{a}\left[\psi_{j}^{*}\right], \mathbf{a}\left[\psi_{j}^{*}\right]\right]_{j, n}=4 & \left(n=0, \ldots, N_{j}-1\right)
\end{array}
$$

for all $j \in \mathbb{N}_{0}$. Hence, $\alpha=\beta=\gamma=\delta=1$. From (5.12) it follows that $\nu=\mu=4$, and thus $K_{j}(n)= \pm 4\left(n \in \mathbb{N}_{0}\right)$. Then the assertion can be obtained by application of theorem 5.3.

\section{Decomposition and reconstruction algorithms}

Now we derive efficient decomposition and reconstruction algorithms. In order to decompose a given function $f_{j+1} \in V_{j+1}\left(j \in \mathbb{N}_{0}\right)$ of the form

$$
f_{j+1}=\sum_{l=0}^{N_{j+1}} \epsilon_{j+1, l} \alpha_{j+1, l}\left(f_{j+1}\right) \sigma_{j+1, l} \varphi_{j+1}
$$

the uniquely determined functions $f_{j} \in V_{j}$ and $g_{j} \in W_{j}$ have to be found such that

$$
f_{j+1}=f_{j}+g_{j}
$$

Assume that the coefficients $\alpha_{j+1, l} \in \mathbb{R}\left(l=0, \ldots, N_{j+1}\right)$ of $f_{j+1}$ or their DCT-I $\left(N_{j+1}+1\right)$ data

$$
\hat{\alpha}_{j+1, k}:=\sum_{l=0}^{N_{j+1}} \epsilon_{j+1, l} \alpha_{j+1, l}\left(f_{j+1}\right) \cos \frac{k l \pi}{N_{j+1}} \quad\left(k=0, \ldots, N_{j+1}\right)
$$

are known. The wanted functions $f_{j} \in V_{j}$ and $g_{j} \in W_{j}$ can be uniquely represented by

$$
f_{j}=\sum_{m=0}^{N_{j}} \epsilon_{j, m} \alpha_{j, m}\left(f_{j}\right) \sigma_{j, m} \varphi_{j}, \quad g_{j}=\sum_{r=0}^{N_{j}-1} \beta_{j, r}\left(g_{j}\right) \sigma_{j+1,2 r+1} \psi_{j}
$$

with unknown coefficients $\alpha_{j, m}\left(f_{j}\right), \beta_{j, r}\left(g_{j}\right) \in \mathbb{R}$. Let $\hat{\alpha}_{j, k}, \tilde{\beta}_{j, s} \in \mathbb{R}$ denote the 
following DCT-I $\left(N_{j}+1\right)$ and DCT-II $\left(N_{j}\right)$ data

$$
\begin{aligned}
\hat{\alpha}_{j, k}:=\sum_{m=0}^{N_{j}} \epsilon_{j, m} \alpha_{j, m}\left(f_{j}\right) \cos \frac{k m \pi}{N_{j}} & \left(k=0, \ldots, N_{j}\right), \\
\tilde{\beta}_{j, s} & :=\sum_{r=0}^{N_{j}-1} \beta_{j, r}\left(g_{j}\right) \cos \frac{(2 r+1) s \pi}{N_{j+1}} \quad\left(s=0, \ldots, N_{j}-1\right) .
\end{aligned}
$$

In order to reconstruct $f_{j+1} \in V_{j+1}\left(j \in \mathbb{N}_{0}\right)$, we have to compute the sum (6.2) with given functions $f_{j} \in V_{j}$ and $g_{j} \in W_{j}$. Assume that $\alpha_{j, m}\left(f_{j}\right), \beta_{j, r}\left(g_{j}\right) \in \mathbb{R}$ in (6.4) or the corresponding DCT data (6.5)-(6.6) are known. Then $f_{j+1} \in V_{j+1}$ can be uniquely represented in the form (6.1).

The decomposition and reconstruction algorithms are based on the following connection between (6.3) and (6.5)-(6.6):

\section{Theorem 6.1}

Assume that for $j \in \mathbb{N}_{0}$

$$
a_{k}\left[\varphi_{j}\right] \neq 0 \quad\left(k=0, \ldots, N_{j}\right) .
$$

For $j \in \mathbb{N}_{0}$, let $f_{j+1} \in V_{j+1}, f_{j} \in V_{j}$ and $g_{j} \in W_{j}$ with (6.1)-(6.6) be given. Then we have

$$
\begin{aligned}
\left(\begin{array}{c}
\hat{\alpha}_{j+1 . r} \\
\hat{\alpha}_{j+1, N_{j+1}-r}
\end{array}\right) & =\mathbf{S}_{j+1}(r)\left(\begin{array}{c}
\hat{\alpha}_{j, r} \\
\tilde{\beta}_{j, r}
\end{array}\right) \quad\left(r=0, \ldots, N_{j}-1\right), \\
\hat{\alpha}_{j+1, N_{j}} & =A_{j+1}\left(N_{j}\right) \hat{\alpha}_{j, N_{j} .}
\end{aligned}
$$

Proof

From (6.4), it follows by lemma 3.1 , (iv) that for all $n \in \mathbb{N}_{0}$

with

$$
a_{n}\left[f_{j}\right]=\hat{\alpha}_{j, n} a_{n}\left[\varphi_{j}\right]
$$

$$
\hat{\alpha}_{j, n}:=\sum_{l=0}^{N_{j}} \epsilon_{j, l} \alpha_{j, l}\left(f_{j}\right) \cos \frac{\ln \pi}{N_{j}} .
$$

Analogously, by (6.1) and (6.3)-(6.6) we have for all $n \in \mathbb{N}_{0}$

$$
a_{n}\left[f_{j+1}\right]=\hat{\alpha}_{j+1, n} a_{n}\left[\varphi_{j+1}\right], \quad a_{n}\left[g_{j}\right]=\tilde{\beta}_{j, n} a_{n}\left[\psi_{j}\right],
$$

where $\hat{\alpha}_{j+1, n}$ is defined similar to $\hat{\alpha}_{j, n}$ and

$$
\bar{\beta}_{j, n}:=\sum_{r=0}^{N_{j}-1} \beta_{j, r}\left(g_{j}\right) \cos \frac{(2 r+1) n \pi}{N_{j+1}} .
$$

Relation (6.2) holds if and only if for all $k \in \mathbb{N}_{0}$

$$
a_{k}\left[f_{j+1}\right]=a_{k}\left[f_{j}\right]+a_{k}\left[g_{j}\right] \text {. }
$$


Using the Chebyshev transformed two-scale relations (4.8) and (5.2), we obtain

$$
\hat{\alpha}_{j+1, k} a_{k}\left[\varphi_{j+1}\right]=\hat{\alpha}_{j, k} A_{j+1}(k) a_{k}\left[\varphi_{j+1}\right]+\tilde{\beta}_{j, k} B_{j+1}(k) a_{k}\left[\varphi_{j+1}\right] .
$$

Analogously, we have for $k=0, \ldots, N_{j+1}$

$$
\begin{aligned}
\hat{\alpha}_{j+1, N_{j+1}-k} a_{k}\left[\varphi_{j+1}\right]= & \hat{\alpha}_{j, N_{j+1}-k} A_{j+1}\left(N_{j+1}-k\right) a_{k}\left[\varphi_{j+1}\right] \\
& +\bar{\beta}_{j, N_{j+1}-k} B_{j+1}\left(N_{j+1}-k\right) a_{k}\left[\varphi_{j+1}\right] .
\end{aligned}
$$

Using the assumption (6.7) and observing that $\hat{\alpha}_{j, N_{j+1}-k}=\hat{\alpha}_{j, k}, \tilde{\beta}_{N_{j+1}-k}=-\tilde{\beta}_{j, k}$ $\left(k=0, \ldots, N_{j+1}\right)$, we obtain the assertion. Note that from (4.7) and lemma 4.3 it follows that $2 \alpha \beta^{-1} \leq A_{j+1}\left(N_{j}\right)^{2} \leq 2 \alpha^{-1} \beta$, i.e. $A_{j+1}\left(N_{j}\right) \neq 0$.

We obtain the following algorithms:

\section{Algorithm 6.2 (Decomposition algorithm)}

Input: $j \in \mathbb{N}_{0}$,

$$
\hat{\alpha}_{j+1, k} \in \mathbb{R}\left(k=0, \ldots, N_{j+1}\right) .
$$

Form for $r=0, \ldots, N_{j}-1$,

$$
\begin{gathered}
\left(\begin{array}{c}
\hat{\alpha}_{j, r} \\
\tilde{\beta}_{j, r}
\end{array}\right):=\mathbf{S}_{j+1}(r)^{-1}\left(\begin{array}{c}
\hat{\alpha}_{j+1, r} \\
\hat{\alpha}_{j+1, N_{j+1}-r}
\end{array}\right), \\
\hat{\alpha}_{j, N_{j}}:=A_{j+1}\left(N_{j}\right)^{-1} \hat{\alpha}_{j+1, N_{j}} .
\end{gathered}
$$

Output: $\hat{\alpha}_{j, r}\left(r=0, \ldots, N_{j}\right)$,

$$
\tilde{\beta}_{j, r}\left(r=0, \ldots, N_{j}-1\right) \text {. }
$$

\section{Algorithm 6.3 (Reconstruction algorithm)}

Input: $j \in \mathbb{N}_{0}$,

$$
\begin{aligned}
& \hat{\alpha}_{j, r} \in \mathbb{R}\left(r=0, \ldots, N_{j}\right), \\
& \tilde{\beta}_{j, r} \in \mathbb{R}\left(r=0, \ldots, N_{j}-1\right) .
\end{aligned}
$$

Form for $r=0, \ldots, N_{j}-1$,

$$
\begin{aligned}
\left(\begin{array}{c}
\hat{\alpha}_{j+1, r} \\
\hat{\alpha}_{j+1, N_{j+1}-r}
\end{array}\right) & :=\mathbf{S}_{j+1}(r)\left(\begin{array}{c}
\hat{\alpha}_{j, r} \\
\tilde{\beta}_{j, r}
\end{array}\right), \\
\hat{\alpha}_{j+1, N_{j}} & :=A_{j+1}\left(N_{j}\right) \hat{\alpha}_{j, N_{j}} .
\end{aligned}
$$

Output: $\hat{\alpha}_{j+1, k}\left(k=0, \ldots, N_{j+1}\right)$. 


\section{Polynomial wavelets}

As the first example, we consider polynomial wavelets on $I$ (see $[6,16]$ ). Set $N_{j}:=2^{j}\left(j \in \mathbb{N}_{0}\right)$. As scaling function $\varphi_{j}$ of level $j$ we use the following function defined by its Chebyshev coefficients

$$
N_{j} a_{n}\left[\varphi_{j}\right]:= \begin{cases}2 & n=0, \ldots, N_{j}-1 \\ 1 & n=N_{j} \\ 0 & n>N_{j}\end{cases}
$$

Then it holds that

$$
\frac{N_{j}}{2} \varphi_{j}=\sum_{k=0}^{N_{j}} \epsilon_{j, k} T_{k} \in \Pi_{N_{j}}
$$

By (7.1), the corresponding bracket product reads as follows

$$
N_{j}^{2}\left[\mathbf{a}\left[\varphi_{j}\right], \mathbf{a}\left[\varphi_{j}\right]\right]_{j, k}= \begin{cases}4 & k=0, \ldots, N_{j}-1, \\ 2 & k=N_{j} .\end{cases}
$$

Using (2.8), we obtain the following interpolation property of $\varphi_{j}$

$$
\varphi_{j}\left(h_{j, l}\right)=\sigma_{j, l} \varphi_{j}(1)=\frac{2}{N_{j}} \sum_{k=0}^{N_{j}} \epsilon_{j, k} \cos \frac{k l \pi}{N_{j}}=2 \delta_{0, l} \quad\left(l=0, \ldots, N_{j}\right) .
$$

By (7.1), the shifted scaling functions $\sigma_{j, k} \varphi_{j}\left(k=0, \ldots, N_{j}\right)$ are contained in $\Pi_{N_{j}}$. Further, these functions $\sigma_{j, k} \varphi_{j}\left(k=0, \ldots, N_{j}\right)$ are modified Lagrange fundamental polynomials with respect to the Gauss-Chebyshev nodes $h_{j, l}\left(l=0, \ldots, N_{j}\right)$, since for $k, l=0, \ldots, N_{j}$ from lemma 3.1, (ii) and (3.1) it follows

$$
\begin{aligned}
\sigma_{j, k} \varphi_{j}\left(h_{j, l}\right) & =\left(\sigma_{j, l} \sigma_{j, k} \varphi_{j}\right)(1) \\
& =\frac{1}{2}\left(\sigma_{j, l+k} \varphi_{j}(1)+\sigma_{j,|l-k|} \varphi_{j}(1)\right) \\
& =\epsilon_{j, k}^{-1} \delta_{k, l} .
\end{aligned}
$$

Figure 1 shows the scaling function $\varphi_{5}$, and figure 2 presents the shifted function $\sigma_{5,16} \varphi_{5}$. The function $\sigma_{j, k} \varphi_{j}\left(k=0, \ldots, N_{j}\right)$ is supported on the whole interval $I$, and has significant values in a small neighbourhood of $h_{j, k}$, if $j$ is large enough.

Let $V_{j}:=S_{j, 0}\left(\varphi_{j}\right)$ be the same space of level $j$. Consequently, by lemma 3.5, (i), the polynomials $\sigma_{j, k} \varphi_{j}\left(k=0, \ldots, N_{j}\right)$ form a basis of $V_{j}$, i.e.,

$$
V_{j}=\Pi_{N_{j}}, \quad \operatorname{dim} V_{j}=N_{j}+1 .
$$

Note that the operator $L_{j}: C(I) \rightarrow V_{j}$ defined by

$$
L_{j} f:=\sum_{k=0}^{N_{j}} \epsilon_{j, k} f\left(h_{j, k}\right) \sigma_{j, k} \varphi_{j} \quad(f \in C(I))
$$




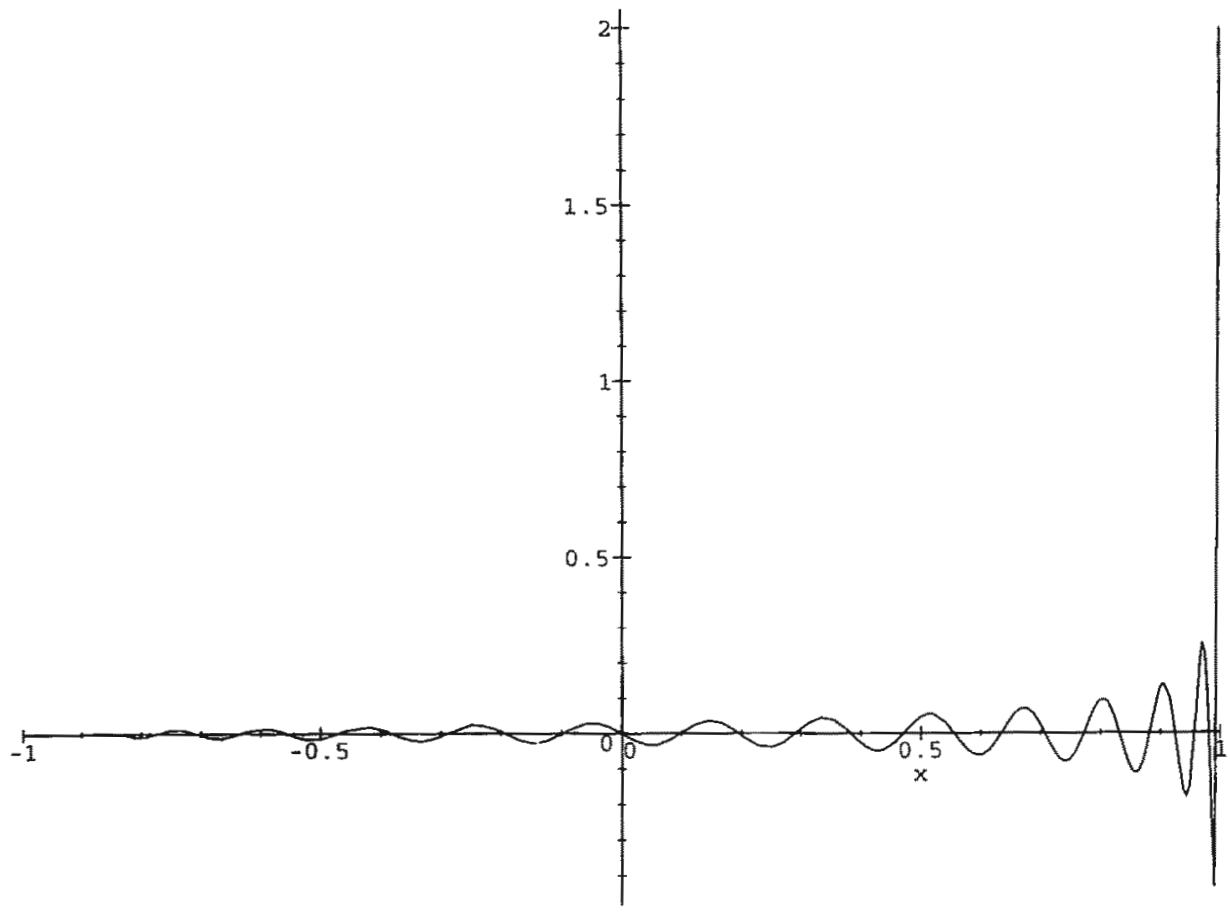

Figure 1.

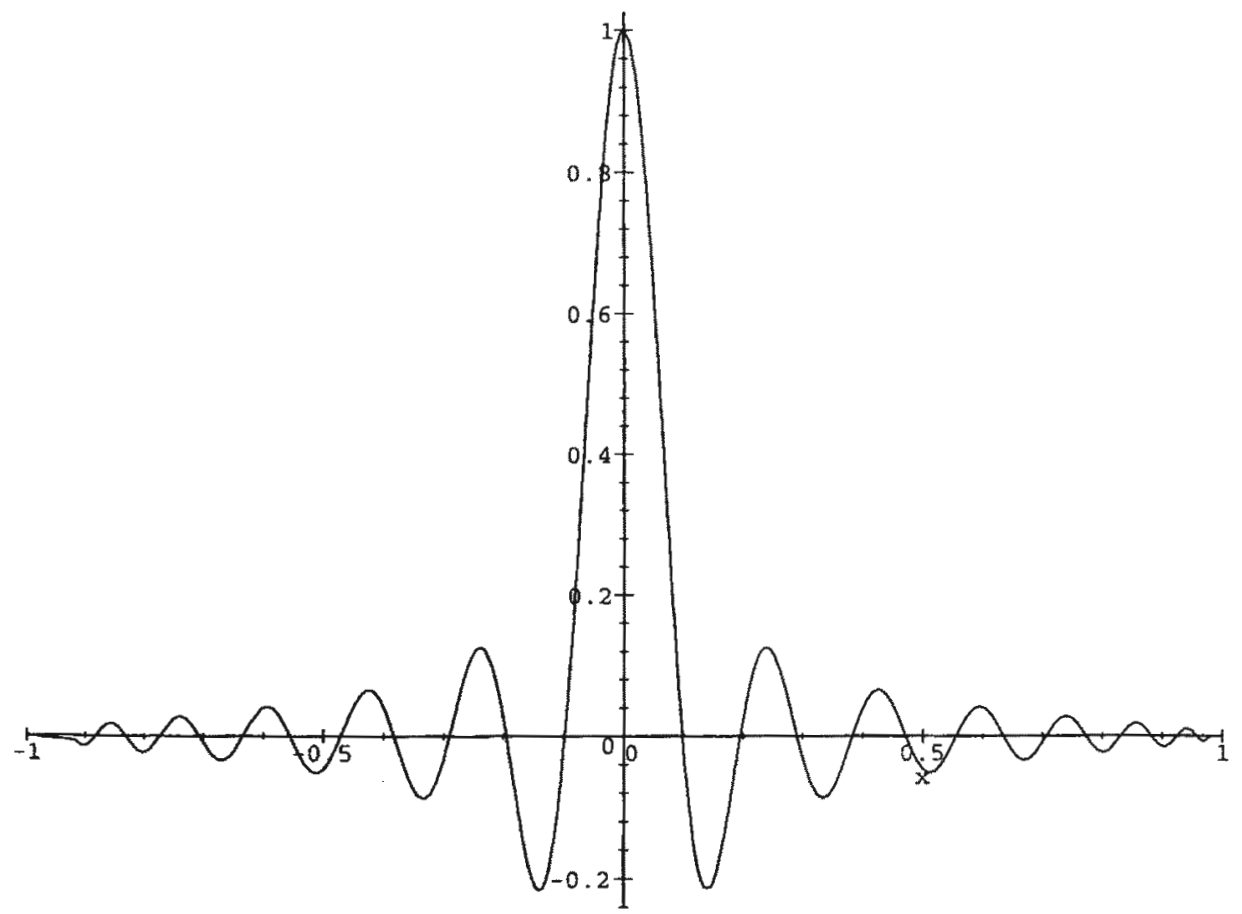

Figure 2. 
is an interpolation operator, which maps $C(I)$ onto $V_{j}$ with the property

$$
L_{j} f\left(h_{j, l}\right)=f\left(h_{j, l}\right) \quad\left(l=0, \ldots, N_{j}\right) .
$$

All sample spaces $V_{j}\left(j \in \mathbb{N}_{0}\right)$ form a multiresolution of $L_{w}^{2}(I)$, where (M3) reads as follows: The systems $\mathcal{B}_{j .0}\left(\left(N_{j} / 2\right)^{1 / 2} \varphi_{j}\right)\left(j \in \mathbb{N}_{0}\right)$ are $L_{w}^{2}(I)$-stable with optimal constants $\alpha=1 / 2$ and $\beta=1$, i.e., for all $j \in \mathbb{N}_{0}$ and for any $\left(\alpha_{j, k}\right)_{k=0}^{N_{j}} \in \mathbb{R}^{N_{j}+1}$ we have the sharp estimate

$$
\frac{1}{2} \sum_{k=0}^{N_{j}} \epsilon_{j, k} \alpha_{j, k}^{2} \leq\left\|\sum_{k=0}^{N_{j}} \epsilon_{j, k} \alpha_{j, k}\left(N_{j} / 2\right)^{1 / 2} \sigma_{j, k} \varphi_{j}\right\|^{2} \leq \sum_{k=0}^{N_{i}} \epsilon_{j, k} \alpha_{j, k}^{2} .
$$

Using (7.1), we find the Chebyshev transformed two-scale relation of $\varphi_{j}$

$$
a_{n}\left[\varphi_{j}\right]=A_{j+1}(n) a_{n}\left[\varphi_{j+1}\right] \quad\left(n \in \mathbb{N}_{0}\right)
$$

with the corresponding two-scale symbol

$$
A_{j+1}(n):= \begin{cases}2 & n=0, \ldots, N_{j}-1, \\ 1 & n=N_{j}, \\ 0 & n=N_{j}+1, \ldots, N_{j+1} .\end{cases}
$$

Let $W_{j}:=V_{j+1} \ominus V_{j}$ be the wavelet space of level $j$. Thus, $\operatorname{dim} W_{j}=N_{j}$. Consider the polynomials $\psi_{j} \in V_{j+1}\left(j \in \mathbb{N}_{0}\right)$ given by their Chebyshev coefficients

$$
N_{j} a_{n}\left[\psi_{j}\right]:= \begin{cases}2 & n=N_{j}+1, \ldots, N_{j+1}-1, \\ 1 & n=N_{j+1}, \\ 0 & \text { otherwise. }\end{cases}
$$

Then the corresponding bracket product reads as follows

$$
N_{j}^{2}\left[\mathbf{a}\left[\psi_{j}\right], \mathbf{a}\left[\psi_{j}\right]\right]_{j+1, k}= \begin{cases}0 & k=0, \ldots, N_{j}, \\ 4 & k=N_{j}+1, \ldots, N_{j+1}-1, \\ 2 & k=N_{j+1} .\end{cases}
$$

The shifted polynomials $\sigma_{j+1,2 r+1} \psi_{j}$ satisfy the interpolation properties

$$
\sigma_{j+1,2 r+1} \psi_{j}\left(h_{j+1,2 s+1}\right)=\delta_{r, s} \quad\left(r, s=0, \ldots, N_{j}-1\right) .
$$

Figure 3 shows the wavelet $\psi_{5}$, and figure 4 presents the shifted wavelet $\sigma_{6,33} \psi_{5}$. The wavelet space $W_{j}$ is a shift-invariant subspace of $L_{w}^{2}(I)$ of type 1 generated by $\psi_{j}$. The systems $\mathcal{B}_{j, 1}\left(\left(N_{j} / 2\right)^{1 / 2} \psi_{j}\right)\left(j \in \mathbb{N}_{0}\right)$ are $L_{w}^{2}(I)$-stable with optimal constants $\gamma=1 / 2$ and $\delta=1$, i.e., for all $j \in \mathbb{N}_{0}$ and for any $\left(\beta_{j, r}\right)_{r=0}^{N_{j}-1} \in \mathbb{R}^{N_{j}}$, we have the sharp estimate

$$
\frac{1}{2} \sum_{r=0}^{N_{j}-1} \beta_{j, r}^{2} \leq\left\|\sum_{r=0}^{N_{j}-1} \beta_{j, r}\left(N_{j} / 2\right)^{1 / 2} \sigma_{j+1,2 r+1} \psi_{j}\right\|^{2} \leq \sum_{r=0}^{N_{j}-1} \beta_{j, r}^{2} .
$$

Using (7.1) and (7.4), we obtain the Chebyshev transformed two-scale relation 


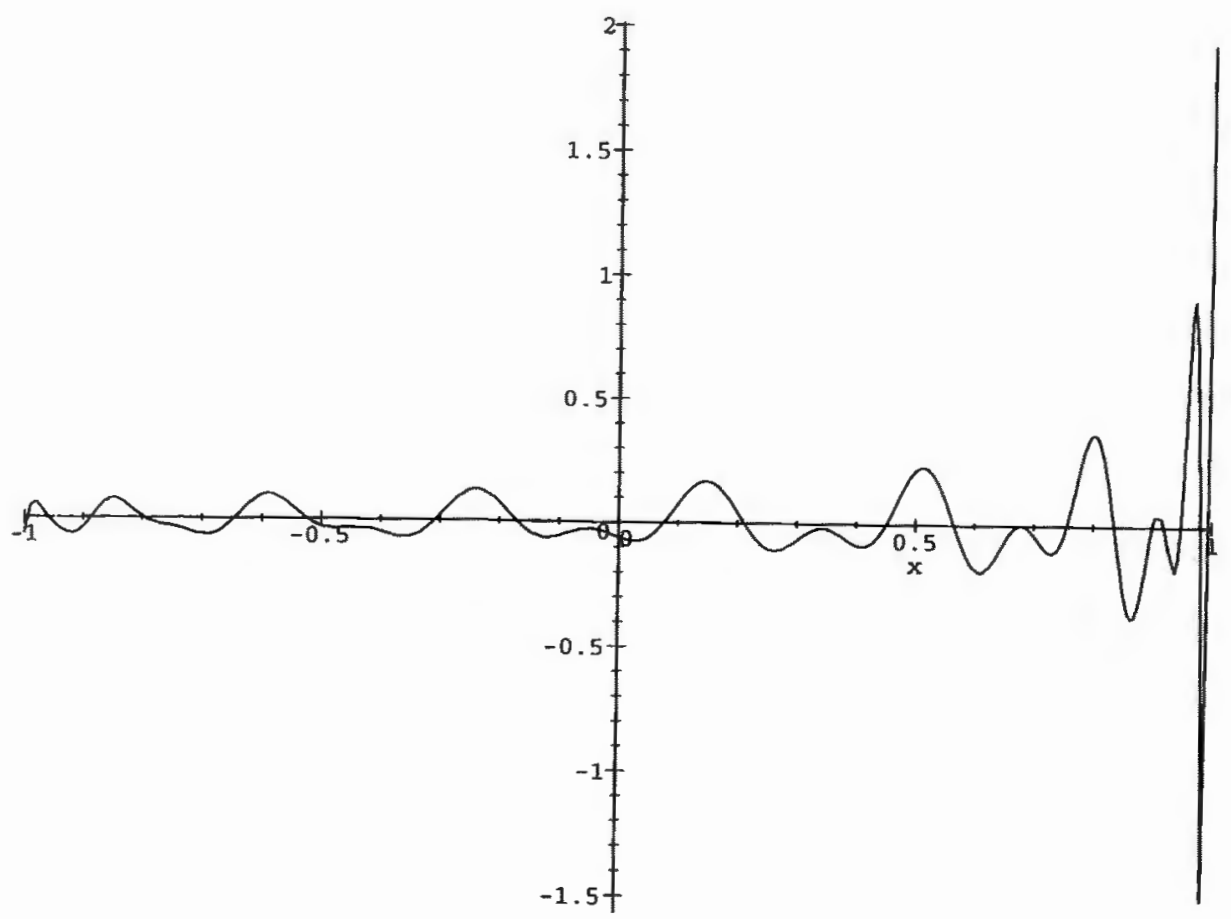

Figure 3.

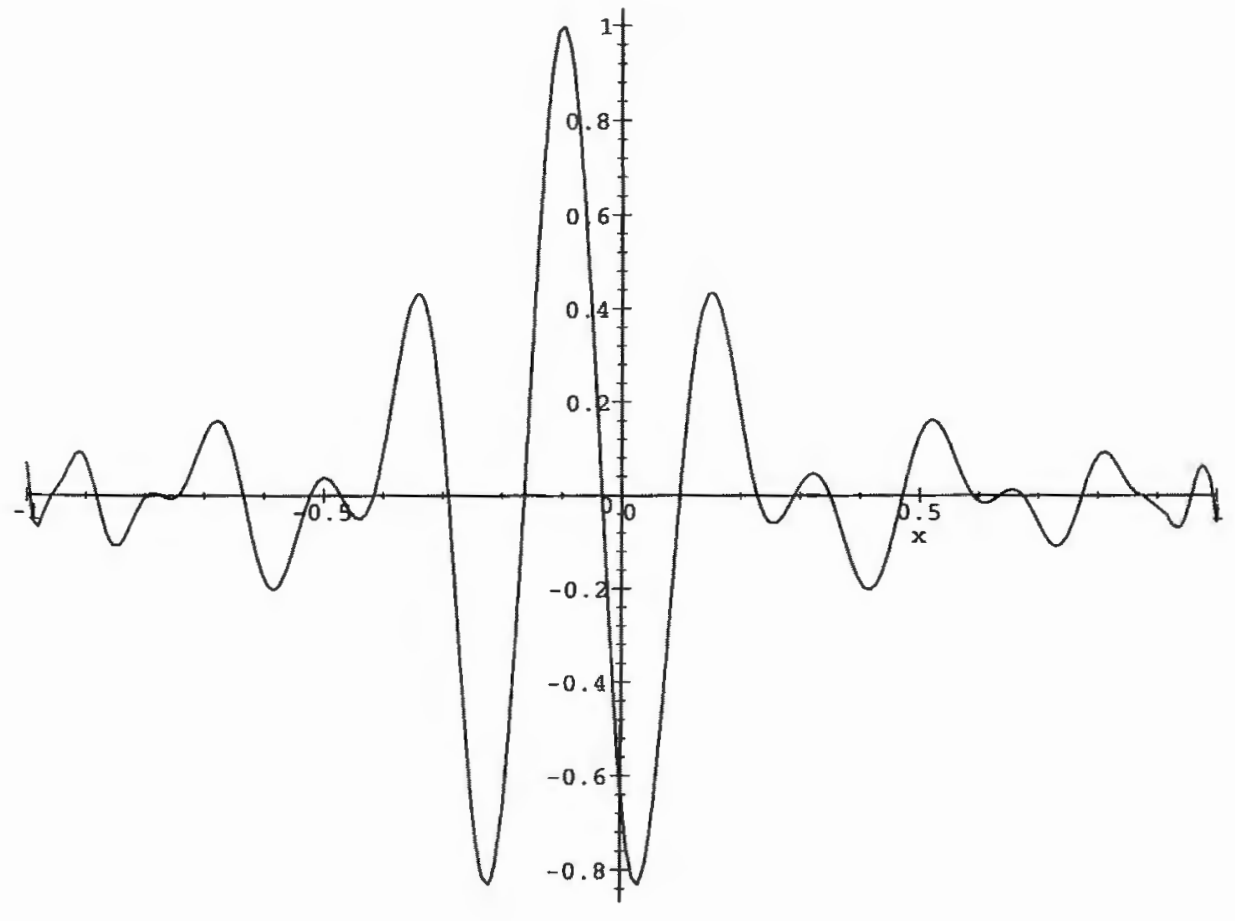

Figure 4. 
of $\psi_{j}$

$$
a_{n}\left[\psi_{j}\right]=B_{j+1}(n) a_{n}\left[\varphi_{i+1}\right] \quad\left(n \in \mathbb{N}_{0}\right)
$$

with the corresponding two-scale symbol

$$
B_{j+1}(n):= \begin{cases}0 & n=0, \ldots, N_{j}, \\ 2 & n=N_{j}+1, \ldots, N_{j+1} .\end{cases}
$$

In the following, we compare the arithmetical complexity of our decomposition algorithm 6.2 for these polynomial wavelets on $I$ with that of the fast decomposition algorithm for linear and cubic spline wavelets on $[0,1]$ proposed in [13]. Let $j \geq 3$. Assume that $2^{j+1}+1$ function values of $f_{j+1} \in V_{j+1}$ are given. The decomposition algorithm for linear spline wavelets in $[0,1]$ needs $6 \cdot 2^{j+1}$ real multiplications in order to compute all wavelet coefficients of $g_{j} \in W_{j}$. For the same problem, the decomposition algorithm for cubic spline wavelets in [13] can be implemented using $14 \cdot 2^{j+1}$ real multiplications. Compared to that, our algorithm 6.2 requires fewer real multiplications up to the level $j=14$.

Now we consider the complete decomposition of $f_{j+1} \in V_{j+1}$. Here we have to determine all coefficients of the related functions in $W_{j}, W_{j-1}, \ldots, W_{3}$ and $V_{3}$. Figure 5 shows the number of needed real multiplications (divided by $2^{j+1}$ ) for the complete decomposition with linear spline wavelets $(\diamond)$, cubic spline wavelets $(\square)$ and polynomial wavelets $(+)$. Our procedure needs fewer real multiplications than the method in [13] for cubic spline wavelets up to level $j=20$. Since a level

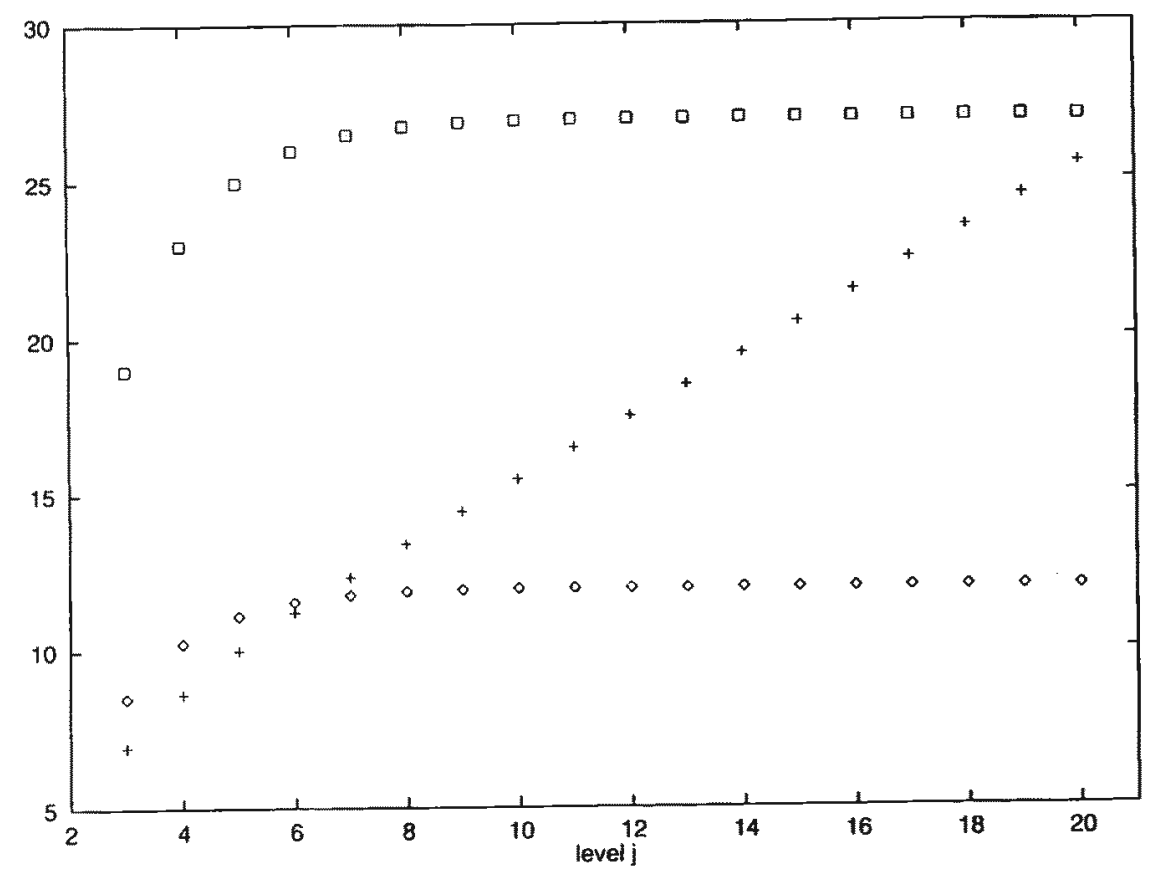

Figure 5. 
$j \in\{7, \ldots, 11\}$ is often used in practice, our algorithm is an interesting alternative to the method in [13].

As a numerical application of the decomposition algorithm 6.2 , we would like to mention that an exact detection of singularities of a given function near the boundary \pm 1 is possible. For example, we consider a linear spline function in order to determine its spline knots. Let $B_{2}$ denote the cardinal linear B-spline. Interpolating the function

$$
f(x):=B_{2}(4 x+3.96) \quad(x \in I)
$$

at level $j=7$ and decomposing $f$, we can observe the singularities at $-0.99,-0.74$ and -0.49 in the corresponding wavelet part of level $j=6$ (see figure 6 ). On the other hand, the decomposition of the function

$$
f(x):=B_{2}(4 x+4) \quad(x \in I)
$$

shows that $f$ has singularities at -0.75 and -0.5 , but not at -1 (see figure 7).

We can generalize the example of polynomial wavelets in a similar manner as done for periodic functions in [14]. Set $N_{j}=d 2^{j}\left(j \in \mathbb{N}_{0}\right)$ with fixed $d \in \mathbb{N}$. Further let for fixed $\lambda \in \mathbb{N}_{0}$

$$
M_{j}:= \begin{cases}1 & j \leq \lambda, \\ 2^{j-\lambda} & j>\lambda\end{cases}
$$

where $3 \leq 2^{\lambda} d$ is fulfilled. Then, $N_{j}+M_{j} \leq N_{j+1}-M_{j+1}$. Let the scaling function $\varphi_{j}$

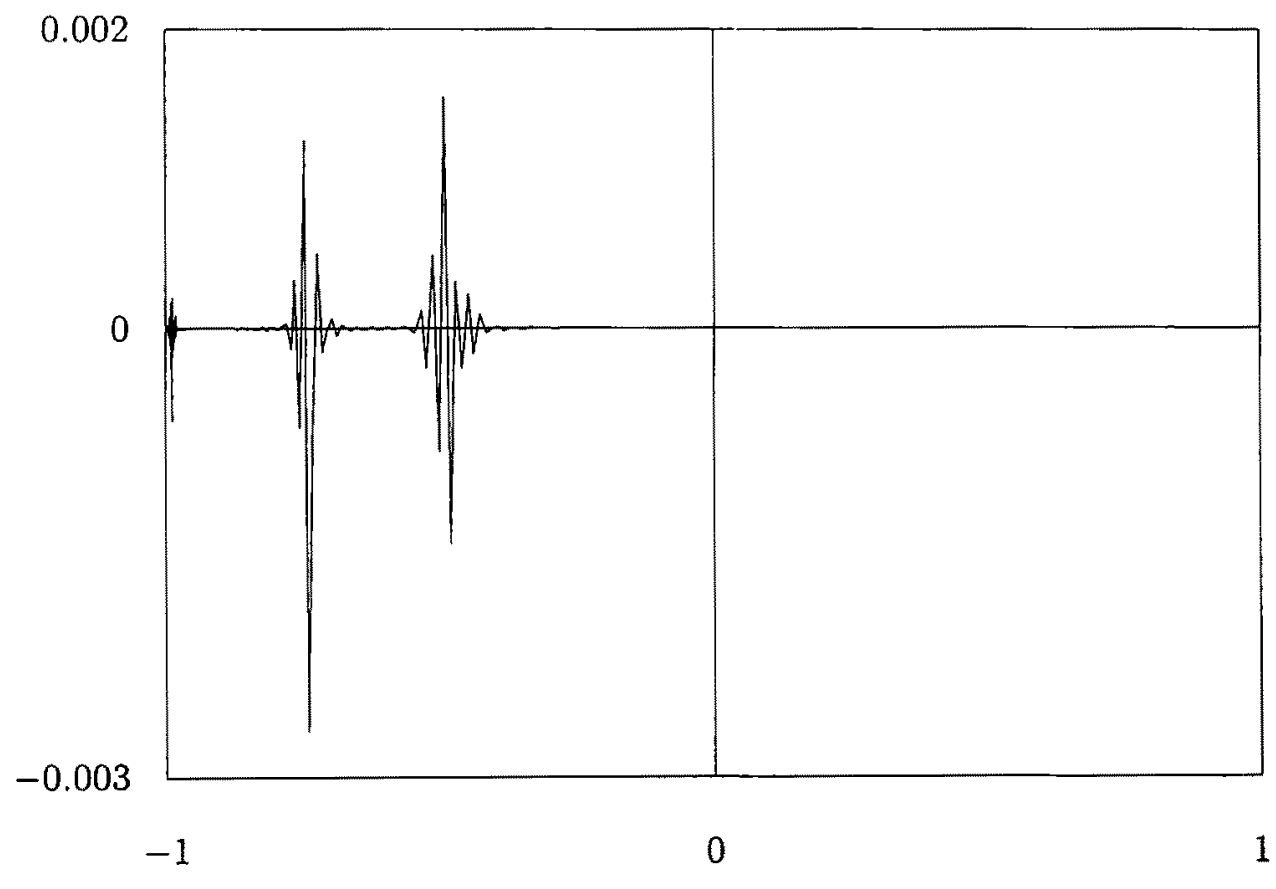

Figure 6. 


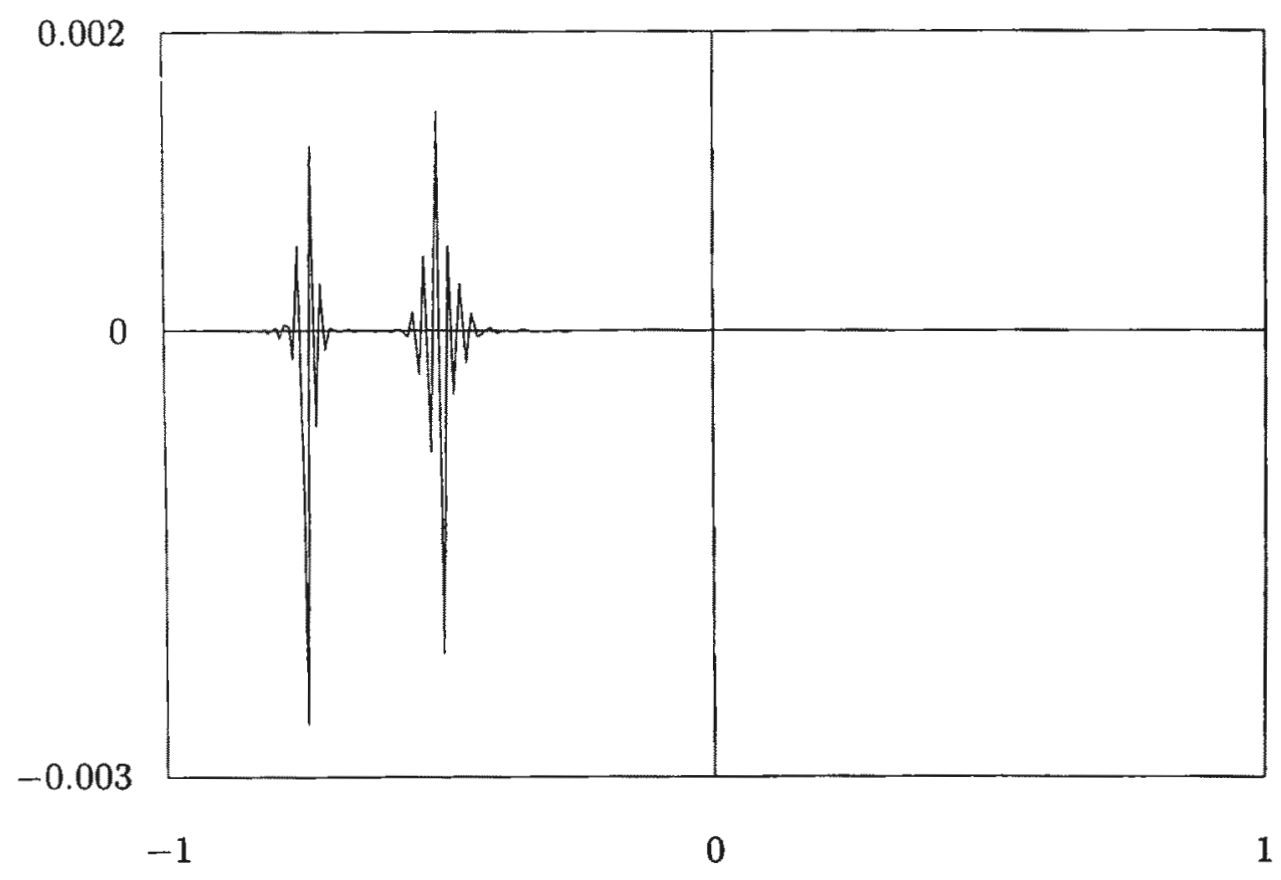

Figure 7.

of level $j$ be given by its Chebyshev coefficients

$$
N_{j} a_{n}\left[\varphi_{j}\right]:= \begin{cases}2 & 0 \leq n \leq N_{j}-M_{j}, \\ \frac{N_{j}+M_{j}-n}{M_{j}} & N_{j}-M_{j}<n<N_{j}+M_{j}, \\ 0 & n \geq N_{j}+M_{j} .\end{cases}
$$

The smaller $\lambda$, the better localized the scaling functions are on $I$. We obtain the same interpolation property of $\varphi_{j}$ as in (7.3). The sample space $V_{j}=S_{j, 0}\left(\varphi_{j}\right)$ can be described by

$$
V_{j}=\Pi_{N_{j}-M_{j}} \oplus \operatorname{span}\left\{\frac{M_{j}+k}{M_{j+1}} T_{N_{j}-k}+\frac{M_{j}-k}{M_{j+1}} T_{N_{j}+k}: k=0, \ldots, M_{j}-1\right\},
$$

i.e., $\Pi_{N_{j}-M_{j}} \subset V_{j} \subset \Pi_{N_{j}+M_{j}-1}$. The corresponding wavelet space $W_{j}\left(j \in \mathbb{N}_{0}\right)$ is of type 1 generated by the polynomial $\psi_{j}:=2 \varphi_{j+1}-\varphi_{j} \in V_{j+1}$ such that

$$
N_{j} a_{n}\left[\psi_{j}\right]= \begin{cases}\frac{n-N_{j}+M_{j}}{M_{j}} & N_{j}-M_{j}<n<N_{j}+M_{j}, \\ \frac{N_{j+1}+M_{j+1}-n}{M_{j+1}} & N_{j}+M_{j} \leq n \leq N_{j+1}-M_{j+1}, \\ 0 & N_{j+1}-M_{j+1}<n<N_{j+1}+M_{j+1},\end{cases}
$$


The shifted polynomials $\sigma_{j+1,2 r+1} \psi_{j}$ also satisfy the interpolation properties

$$
\sigma_{j+1,2 r+1} \psi_{j}\left(h_{j+1,2 s+1}\right)=\delta_{r, s} \quad\left(r, s=0, \ldots, N_{j}-1\right) .
$$

For the Chebyshev transformed two-scale relations of $\varphi_{j}$ and $\psi_{j}$, we obtain the two-scale symbols

$$
A_{j+1}(n):= \begin{cases}2 & 0 \leq n \leq N_{j}-M_{j}, \\ \frac{N_{j}+M_{j}-n}{M_{j}} & N_{j}-M_{j}<n<N_{j}+M_{j}, \\ 0 & N_{j}+M_{j} \leq n \leq N_{j+1}\end{cases}
$$

and

$$
B_{j+1}(n):= \begin{cases}0 & 0 \leq n \leq N_{j}-M_{j}, \\ \frac{n-N_{j}+M_{j}}{M_{j}} & N_{j}-M_{j}<n<N_{j}+M_{j}, \\ 2 & N_{j}+M_{j} \leq n \leq N_{j+1} .\end{cases}
$$

\section{Transformed spline wavelets}

In principle, the following is obtained by transferring the construction of [12] onto the interval. Let $m \in \mathbb{N}$ be a fixed even number and let $M_{m}$ be the centered B-spline of order $m$ with the knots $-m / 2+k(k=0, \ldots, m)$. Set $N_{j}:=2^{j}$ $\left(j \in \mathbb{N}_{0}\right)$. Further, let $\tilde{M}_{m, j}\left(j \in \mathbb{N}_{0}\right)$ be the $2 \pi$-periodization of $M_{m}\left(N_{j} \cdot \pi\right)$, i.e.

$$
\tilde{M}_{m, j}:=\sum_{i=-\infty}^{\infty} M_{m}\left(N_{j} \cdot / \pi-N_{j+1} l\right)
$$

From $\tilde{M}_{m, j}=\tilde{M}_{m, j}(-\cdot)$ it follows that $\tilde{M}_{m, j} \in L_{2 \pi, 0}^{2}$. The Fourier cosine coefficients of $\tilde{M}_{m, j}$ read as follows

$$
a_{n}\left(\tilde{M}_{m, j}\right)=\frac{1}{N_{j}} \hat{M}_{m}\left(n \pi / N_{j}\right)=\frac{1}{N_{j}}\left(\operatorname{sinc} \frac{n \pi}{N_{j+1}}\right)^{m} \quad\left(n \in \mathbb{N}_{0}\right) .
$$

Now restricting $\tilde{M}_{m, j}$ on $[0, \pi]$, we choose as scaling function $\varphi_{j}:=\tilde{M}_{m, j}(\arccos )$, that is, $\varphi_{j}$ has the Chebyshev coefficients

$$
N_{j} a_{n}\left[\varphi_{j}\right]=\hat{M}_{m}\left(n \pi / N_{j}\right)=\left(\operatorname{sinc} \frac{n \pi}{N_{j+1}}\right)^{m} \quad\left(n \in \mathbb{N}_{0}\right) .
$$

For the two-scale symbol of $\varphi_{j}$ we find

$$
A_{j+1}(n)=2\left(\cos \frac{n \pi}{N_{j+2}}\right)^{m} \quad\left(n \in \mathbb{N}_{0}\right)
$$


Let the sample spaces $V_{j}$ be generated by $\varphi_{j}$, i.e. $V_{j}:=S_{j, 0}\left(\varphi_{j}\right)\left(j \in \mathbb{N}_{0}\right)$. Then by

$$
\bigcup_{j=0}^{\infty} \operatorname{supp} \mathbf{a}\left[\varphi_{i}\right]=\mathbb{N}_{0}
$$

it follows that condition (M2) is satisfied.

For the bracket product we obtain by Poisson summation formula

$$
\begin{aligned}
N_{j}^{2}\left[\mathbf{a}\left[\varphi_{j}\right], \mathbf{a}\left[\varphi_{j}\right]\right]_{j, n} & =\sum_{l=0}^{\infty}\left(\hat{M}_{m}\left(\frac{\left(N_{j+1} l+n\right) \pi}{N_{j}}\right)^{2}+\hat{M}_{m}\left(\frac{\left(N_{j+1}(l+1)-n\right) \pi}{N_{j}}\right)^{2}\right) \\
& =\sum_{l=-\infty}^{\infty} \hat{M}_{2 m}\left(2 \pi l+n \pi / N_{j}\right) \\
& =\sum_{k=-\infty}^{\infty} M_{2 m}(k) e^{-i n k \pi / N_{j}}
\end{aligned}
$$

and hence

$$
N_{j}^{2}\left[\mathbf{a}\left[\varphi_{j}\right], \mathbf{a}\left[\varphi_{j}\right]\right]_{j, n}=\Phi_{2 m}\left(e^{-i n \pi / N_{j}}\right)
$$

with the well-known Euler-Frobenius polynomial

$$
\Phi_{2 m}(z):=\sum_{k=-\infty}^{\infty} M_{2 m}(k) z^{k} \quad(z \in \mathbb{C},|z|=1) .
$$

The systems $\mathcal{B}_{j, 0}\left(\left(N_{j} / 2\right)^{1 / 2} \varphi_{j}\right)\left(j \in \mathbb{N}_{0}\right)$ are $L_{w}^{2}(I)$-stable, since we have

$$
\alpha \leq \frac{N_{j}^{2}}{4}\left[\mathbf{a}\left[\varphi_{j}\right], \mathbf{a}\left[\varphi_{j}\right]\right]_{j, n} \leq \beta \quad\left(j \in \mathbb{N}_{0}\right)
$$

with

$$
4 \alpha=\Phi_{2 m}(-1)=\frac{2^{2 m}\left(2^{2 m}-1\right)}{(2 m) !}\left|B_{2 m}\right|, \quad 4 \beta=\Phi_{2 m}(1)=1,
$$

where $B_{2 m}$ denotes the $(2 m)$ th Bernoulli number. Observe that we have found the same constants $\alpha, \beta$ as in the case of the multiresolution generated by cardinal splines of order $m$ (see [12]). Note that different scaling factors of scaling functions are used in [12].

Let the wavelet $\psi_{j}$ be defined by its Chebyshev coefficients

$$
a_{n}\left[\psi_{j}\right]:=2\left(\sin \frac{n \pi}{N_{j+2}}\right)^{m} \Phi_{2 m}\left(-e^{-i n \pi / N_{j+1}}\right) a_{n}\left[\varphi_{j+1}\right] \quad\left(n \in \mathbb{N}_{0}\right)
$$

i.e., $\psi_{j}$ possesses the two-scale symbol

$$
B_{j+1}(n)=2\left(\sin \frac{n \pi}{N_{j+2}}\right)^{m} \Phi_{2 m}\left(-e^{-i n \pi / N_{j+1}}\right) \quad\left(n \in \mathbb{N}_{0}\right) .
$$

By definition it is clear that $\psi_{j} \in V_{j+1}$. In order to show that $W_{j}=S_{j, 1}\left(\psi_{j}\right)$, we 
have to check the orthogonality $V_{j} \perp S_{j, 1}\left(\psi_{j}\right)$ and the $L_{w}^{2}(I)$-stability of $\mathcal{B}_{j, 1}\left(\left(N_{j} / 2\right)^{1 / 2} \psi_{j}\right)\left(j \in \mathbb{N}_{0}\right)$. Since $m$ is even, we easily observe that (5.5) is satisfied for the two-scale symbols $A_{j+1}(n), B_{j+1}(n)$ above. Furthermore, inserting $B_{j+1}(n)$ into (5.4) we obtain for $n=0, \ldots, N_{j}-1$ by lemma 4.3

$$
N_{j}^{2}\left[\mathbf{a}\left[\psi_{j}\right], \mathbf{a}\left[\psi_{j}\right]\right]_{j, n}=\Phi_{2 m}\left(e^{-i n \pi / N_{j}}\right) \Phi_{2 m}\left(e^{-i n \pi / N_{j+1}}\right) \Phi_{2 m}\left(-e^{-i n \pi / N_{j+1}}\right) .
$$

In particular, we obtain $L_{w}^{2}(I)$-stability of $\mathcal{B}_{j, 1}\left(\left(N_{j} / 2\right)^{1 / 2} \psi_{j}\right)$ with the constants

$$
\begin{aligned}
4 \gamma & =\min \left\{\Phi_{2 m}(z) \Phi_{2 m}(-z) \Phi_{2 m}\left(z^{2}\right): z \in \mathbb{C},|z|=1\right\} \\
& =\Phi_{2 m}(-1) \Phi_{2 m}(-i) \Phi_{2 m}(i)>0, \\
4 \delta & =\max \left\{\Phi_{2 m}(z) \Phi_{2 m}(-z) \Phi_{2 m}\left(z^{2}\right): z \in \mathbb{C},|z|=1\right\}<1 .
\end{aligned}
$$

Observe that these constants are the same as the constants found for the wellknown cardinal Chui-Wang wavelet (cf. [12]). Note that different scaling factors of wavelets are used in [12]. In contrast with polynomial wavelets, the shifted scaling functions and wavelets are supported on small subintervals of $I$.

Finally, we will consider the connection of the wavelet $\psi_{j}$ above with the cardinal Chui-Wang wavelet $w_{m}$ given by its Fourier transform

$$
\hat{w}_{m}(u):=\left(-e^{-i u / 2}\right)^{m-1}\left(\frac{1-e^{-i u / 2}}{2}\right)^{m} \Phi_{2 m}\left(-e^{-i u / 2}\right)\left(\frac{1-e^{-i u / 2}}{i u / 2}\right)^{m} \quad(u \in \mathbb{R} \backslash\{0\}) .
$$

Let $\tilde{\psi}_{j}$ be the $2 \pi$-periodization of $\tilde{w}_{m}\left(N_{j} \cdot\right)$ with

$$
\tilde{w}_{m}:=(-1)^{m / 2+1}\left(w_{m}(\cdot / \pi+m)+w_{m}(-\cdot / \pi+m)\right),
$$

i.e.,

$$
\tilde{\psi}_{j}:=\sum_{l=-\infty}^{\infty} \tilde{w}_{m}\left(N_{j} \cdot-N_{j+1} \pi l\right)
$$

Then $\tilde{\psi}_{j} \in L_{2 \pi, 0}^{2}$. The Fourier cosine coefficients read

$$
\begin{aligned}
a_{n}\left(\tilde{\psi}_{j}\right) & =\frac{2}{N_{j}} \cos \frac{n \pi}{N_{j+1}}\left(\sin \frac{n \pi}{N_{j+2}}\right)^{m} \Phi_{2 m}\left(-e^{-i n \pi / N_{j+1}}\right)\left(\operatorname{sinc} \frac{n \pi}{N_{j+2}}\right)^{m} \\
& =2 \cos \frac{n \pi}{N_{j+1}}\left(\sin \frac{n \pi}{N_{j+2}}\right)^{m} \Phi_{2 m}\left(-e^{-i n \pi / N_{j+1}}\right) a_{n}\left[\varphi_{j+1}\right]
\end{aligned}
$$

Comparing with the Chebyshev coefficients of $\psi_{j}$ we find for the restriction of $\bar{\psi}_{j}$ on $[0, \pi]$ :

$$
\sigma_{j+1,1} \psi_{j}=\tilde{\psi}_{j}(\arccos )
$$




\section{Acknowledgements}

The research of G. Plonka and M. Tasche was supported by the Deutsche Forschungsgemeinschaft. The authors wish to thank D. Potts for useful discussions and numerical experiments and the unknown referees for their hints.

\section{References}

[1] G. Baszenski and M. Tasche, Fast DCT-algorithms, interpolating wavelets and hierarchical bases, in: Curves and Surfaces II, eds. P.J. Laurent, A. Le Méhauté and L.L. Schumaker (A.K. Peters, Boston, 1994) pp. 37-50.

[2] P.L. Butzer and R.L. Stens, The operational properties of the Chebyshev transform. I: General properties, Funct. Approx. Comment. Math. 5 (1977) 129-160.

[3] C.K. Chui and E. Quak, Wavelets on a bounded interval, in: Numerical Methods of Approximation Theory, eds. D. Braess and L.L. Schumaker (Birkhäuser, Basel, 1992) pp. 53-75.

[4] A. Cohen, I. Daubechies and P. Vial, Wavelets on the interval and fast wavelet transforms, Appl. Comp. Harmonic Anal. 1 (1993) 54-81.

[5] C. de Boor, R.A. DeVore and A. Ron, On the construction of multivariate (pre)wavelets, Constr. Approx. 9 (1993) 787-806.

[6] T. Kilgore and J. Prestin, Polynomial wavelets on the interval, Constr. Approx., to appear.

[7] Y.W. Koh, S.L. Lee and H.H. Tan, Periodic orthogonal splines and wavelets, Appl. Comp. Harmonic Anal. 22 (1995) 201-218.

[8] Y. Meyer, Ondelettes due l'intervalle, Rev. Mat. Iberoamer. 7 (1992) 115-133.

[9] C.A. Micchelli and Y. Xu, Using the matrix refinement equation for the construction of wavelets on invariant sets, Appl. Comp. Harmonic Anal. 1 (1994) 391-401.

[10] S. Paszkowski, Numerical Applications of Chebyshev Polynomials and Series (Nauka, Moscow, 1983), in Russian.

[11] G. Plonka and M. Tasche, A unified approach to periodic wavelets, in: Wavelets: Theory, Algorithms, and Applications, eds. C.K. Chui, L. Montefusco and L. Puccio (Academic Press, San Diego, 1994) pp. 137-151.

[12] G. Plonka and M. Tasche, On the computation of periodic spline wavelets, Appl. Comp. Harmonic Anal. 2 (1995) 1-14.

[13] E. Quak and N. Weyrich, Decomposition and reconstruction algorithms for spline wavelets on a bounded interval, Appl. Comp. Harmonic Anal. 1 (1994) 217-231.

[14] K. Selig, Trigonometric wavelets for time-frequency-analysis, in: Approximation Theory, Wavelets and Applications, ed. S.P. Singh (Kluwer Academic, Dordrecht, 1995) pp. 453-464.

[15] M. Tasche, Fast algorithms for discrete Chebyshev--Vandermonde transforms and applications, Numer. Algor. 5 (1993) 453-464.

[16] M. Tasche, Polynomial wavelets on $[-1,1]$, in: Approximation Theory, Wavelets and Applications, ed. S.P. Singh (Kluwer Academic, Dordrecht, 1995) pp. 497-512. 